\title{
MULTIDISCIPLINARY APPROACH IN THE DIAGNOSIS AND MANAGEMENT OF PHILADELPHIA CHROMOSOME-NEGATIVE MYELOPROLIFERATIVE NEOPLASMS
}

Ph.D. dissertation

Imelda Marton M.D.

2016 


\title{
Multidisciplinary approach in the diagnosis and management of Philadelphia chromosome-negative myeloproliferative neoplasms
}

\author{
Imelda Marton M.D. \\ Ph.D. dissertation
}

\author{
Supervisors: \\ Professor Zita Borbényi M.D., Ph.D., \\ Professor Attila Nemes M.D.,Ph.D., D.Sc
}

Haematology Division,

2nd Department of Medicine and Cardiology Centre,

University of Szeged

SZEGED

2016 


\section{LIST OF PUBLICATIONS RELATED TO THE THESIS}

I. Éva Pósfai ${ }^{*}$ Imelda Marton ${ }^{*}$ Zita Borbényi, Attila Nemes: Myocardial infarction as a thrombotic complication of essential thrombocythaemia and polycythaemia vera The Anatolian Journal of Cardiology.2016 IF 0.927 - accepted for publication -

Co-authors Éva Pósfai and Imelda Marton contributed equally to this work and are equal in status.

II. Éva Pósfai * Imelda Marton * Zsuzsanna Kiss-László, Balázs Kotosz, Márta Széll, Zita Borbényi: Thrombosis and risk factors in female patients with a rare acquired thrombophilia: chronic myeloproliferative disorder - polycythaemia vera and essential thrombocythaemia. Eur Rev Med Pharmacol Sci 2014;18(24): 3810-3818. IF 0.988 Co-authors Éva Pósfai and Imelda Marton contributed equally to this work and are equal in status.

III. Imelda Marton, László Krenács, Enikő Bagdi, Annamária Bakos, Judit Demeter, Zita Borbényi: Clinical and molecular diagnostic evaluation of systemic mastocytosis in the South-Eastern Hungarian population between 2001-2013 - a single centre experience. Pathol Oncol Res. 2016 Apr;22(2):293-9. IF 1.806

IV. Imelda Marton, Éva Pósfai, Zita Borbényi, Csaba Bödör, Papp Gergely, Demeter Judit, Irma Korom, Erika Varga, Zsuzsanna Bata-Csörgő: Therapeutic challenge during the long-term follow-up of a patient with indolent systemic mastocytosis with extensive cutaneous involvement. European Review for Medical and Pharmacological Sciences. 2015 May;19(9):1607-9. IF 0.988

V. Attila Nemes, Imelda Marton, Péter Domsik, Anita Kalapos, Éva Pósfai, Szabolcs Modok, Zita Borbényi, Tamás Forster: Characterization of left atrial dysfunction in hypereosinophilic syndrome - Insights from the Motion analysis of the heart and great vessels by three-dimensional speckle tracking echocardiography in pathological cases (MAGYAR-Path) Study. Revista Portuguesa de Cardiologia. 2016- IF 0.454 accepted for publication -

VI. Imelda Marton, Éva Pósfai, János Kristóf Annus, Zita Borbényi, Attila Nemes, László Vécsei, Erika Vörös: Watershed infarction in hypereosinophilic syndrome: a diagnostic dilemma in FIP1L1-PDGFR alpha-associated myeloid neoplasm and overview of the relevant literature. Ideggyogy Sz. 2015 May 30;68(5-6):212-6. IF 0.348

VII. Attila Nemes, Anita Kalapos, Péter Domsik, Imelda Marton, Zita Borbenyi, Tamás Forster: Three-dimensional speckle-tracking echocardiography in Loeffler endocarditis: case report from the MAGYAR-Path Study. Herz. 2014 Sep;39(6):722-4. IF 0,7 


\section{LIST OF ABBREVIATIONS}

\begin{tabular}{|l|l|}
\hline 2D & two-dimensional \\
\hline 3D & three-dimensional \\
\hline 3DSTE & three-dimensional speckle-tracking echocardiography \\
\hline AAEF & active atrial emptying fraction \\
\hline AASV & active atrial stroke volume \\
\hline AEC & absolute eosinophil count \\
\hline AMI & acute myocardial infarction \\
\hline AML & acute myeloid leukaemia \\
\hline AP2CH & apical two-chamber (view) \\
\hline AP4CH & apical four-chamber (view) \\
\hline ASA & acetylsalicylic acid \\
\hline ASM & aggressive systemic mastocytosis \\
\hline B-ALL & B-cell acute lymphoblastic leukaemia \\
\hline CEL/HES & chronic eosinophilic leukaemia/hypereosinophilic syndrome \\
\hline CEL-NOS & chronic eosinophilic leukaemia - not otherwise specified \\
\hline CM & cutaneous mastocytosis \\
\hline CML & chronic myeloid leukaemia \\
\hline CNS & central nervous system \\
\hline CS & circumferential strain \\
\hline CV & cardiovascular \\
\hline DM & diabetes mellitus \\
\hline DVT & deep vein thrombosis \\
\hline ECLAP & European Collaboration Study on Low-dose Aspirin in Polycythemia \\
\hline ECNM & European Competence Network on Mastocytosis \\
\hline EDTA & ethylenediaminetetraacetic acid \\
\hline EDV & end-diastolic volume \\
\hline est LV & estimated left ventricular mass \\
\hline MASS & ejection fraction \\
\hline ESV & end-systolic volume \\
\hline ET & \\
\hline EF & essential thrombocythaemia \\
\hline
\end{tabular}




\begin{tabular}{|l|l|}
\hline F & female \\
\hline FFPE & formaldehyde-fixed and paraffin-embedded \\
\hline FGFR1 & fibroblast growth factor receptor 1 \\
\hline FIP1L1 & Fip1-like-1 \\
\hline FISH & fluorescence in situ hybridization \\
\hline Hb & haemoglobin \\
\hline Hct & haematocrit \\
\hline HES & hypereosinophilic syndrome \\
\hline HSC & haematopoietic stem cell \\
\hline ICD & International Classification of Diseases \\
\hline IFN-alpha & interferon-alpha \\
\hline iHES & idiopathic hypereosinophilic syndrome \\
\hline ISM & indolent systemic mastocytosis \\
\hline JAK2 & Janus kinase 2 \\
\hline LA & left atrial \\
\hline LAD & left anterior descending (coronary artery) \\
\hline LCX & left circumflex (coronary artery) \\
\hline LIMA & left internal mammary artery \\
\hline LM & left main (coronary artery) \\
\hline LS & longitudinal strain \\
\hline LV & left ventricle / left ventricular \\
\hline M & male \\
\hline MCA & middle cerebral artery \\
\hline MCD & mast cell disease \\
\hline MCL & mast cell leukaemia \\
\hline MCS & mast cell sarcoma \\
\hline MDS & myelodysplastic syndrome \\
\hline MF & myelofibrosis \\
\hline MM & multiple myeloma \\
\hline MPD & myeloproliferative disease / disorder \\
\hline MPN & myeloproliferative neoplasm \\
\hline NSTEMI & non-ST-segment elevation myocardial infarction \\
\hline PAD & peripheral arterial disease \\
\hline
\end{tabular}




\begin{tabular}{|c|c|}
\hline PAEF & passive atrial emptying fraction \\
\hline PASV & passive atrial stroke volume \\
\hline PCA & posterior cerebral artery \\
\hline PCI & percutaneous coronary intervention \\
\hline PCR & polymerase chain reaction \\
\hline PDGFRA /B & platelet-derived growth factor receptor alpha/beta \\
\hline $\mathrm{PE}$ & pulmonary embolism \\
\hline $\mathrm{Ph}$ & Philadelphia chromosome \\
\hline PLT & platelet \\
\hline PMF & primary myelofibrosis \\
\hline PTCL & peripheral T-cell lymphoma \\
\hline PV & polycythaemia vera \\
\hline PVSG & Polycythemia Vera Study Group \\
\hline RBC & red blood cell \\
\hline $\mathrm{RC}$ & right coronary (artery) \\
\hline $\mathrm{RS}$ & radial strain \\
\hline RT-PCR & real-time polymerase chain reaction \\
\hline SM & systemic mastocytosis \\
\hline SM-AHNMD & $\begin{array}{l}\text { systemic mastocytosis with an associated clonal haematological non-mast } \\
\text { cell lineage disease }\end{array}$ \\
\hline SV & stroke volume \\
\hline SVG & saphenous vein graft \\
\hline TAEF & total atrial emptying fraction \\
\hline TASV & total atrial stroke volume \\
\hline TIA & transient ischaemic attack \\
\hline TTE & transthoracal echocardiography \\
\hline UP & urticaria pigmentosa \\
\hline VBI & vertebrobasilar insufficiency \\
\hline $\mathrm{V}_{\max }$ & maximum (left atrial) volume \\
\hline$V_{\min }$ & minimum (left atrial) volume \\
\hline $\mathrm{V}_{\text {preA }}$ & (left atrial) volume before atrial contraction \\
\hline WBC & white blood cell \\
\hline WHO & World Health Organization \\
\hline
\end{tabular}




\section{TABLE OF CONTENTS}

1. INTRODUCTION..........................................................................................................

2. AIMS

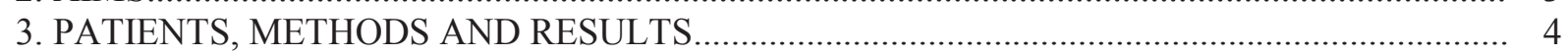

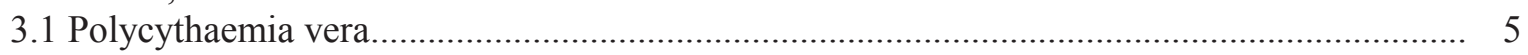

3.1.1 General consideration and background of the polycythaemia vera study....................... 5

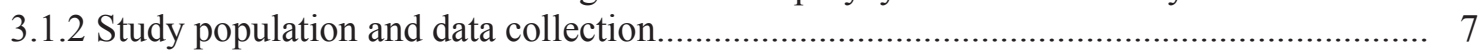

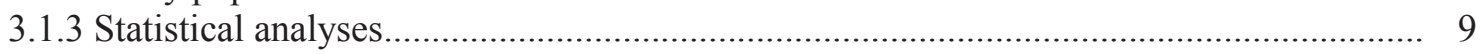

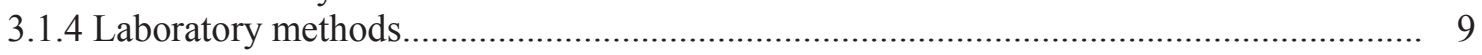

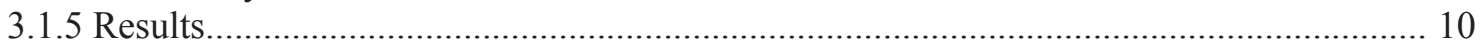

Thrombotic events prior to and during follow-up and the main clinical characterteristics of polycythaemia vera patients with or without thrombotic complications in the follow-up period................................................................... 10

Cardiovascular and cerebrovascular complications.......................................... 12

The contribution of cardiovascular risk factors to subsequent thrombotic

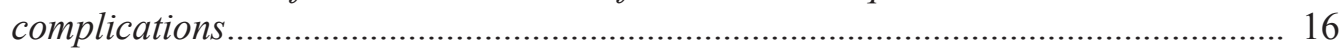

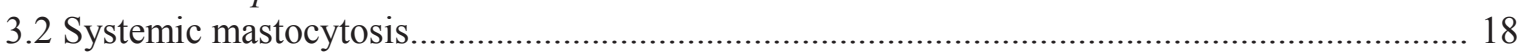

3.2.1 General considerations and background of the systemic mastocytosis study.................... 18

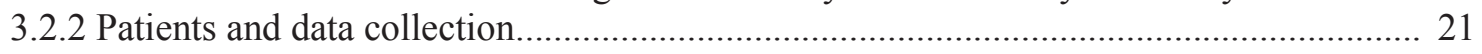

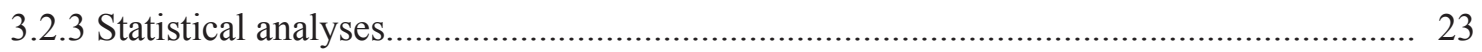

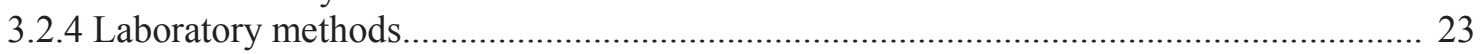

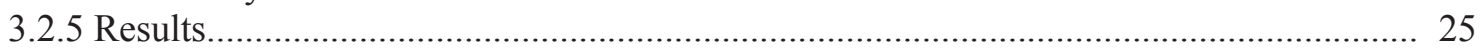

Clinicopathological findings along with bone marrow histological features, molecular characteristics, and laboratory parameters at presentation in a large cohort of systemic mastocytosis patients................................................... 25

Frequency of KIT D816V mutation.................................................................. 26

Life expectancy in systemic mastocytosis.............................................................. 26

Cumulative incidence of systemic mastocytosis..................................................... 28

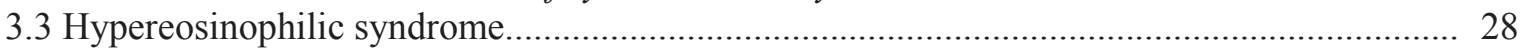

3.3.1 General considerations, background of the hypereosinophilic syndrome study............. 28

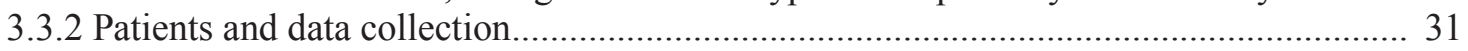

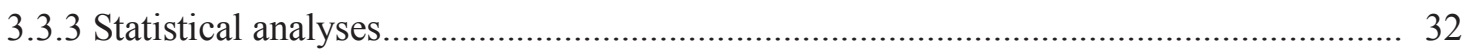

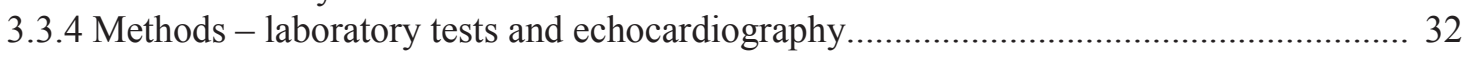

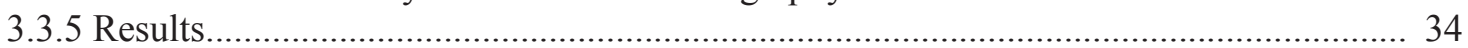

Comparison of left atrial volumetric, volume-based functional, and strain parameters obtained by three-dimensional speckle-tracking echocardiography, a novel, non-invasive clinical tool for volumetric and strain analysis, between patients with hypereosinophilic syndrome and matched controls........................... 34 Presentation of left ventricular rotational mechanics through a unique case of hypereosinophilic syndrome with Loeffler's endocarditis by means of the novel

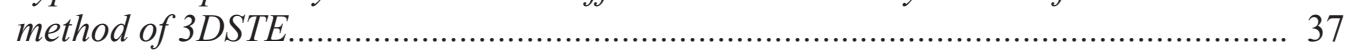

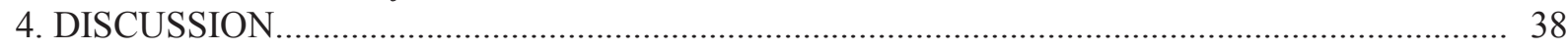

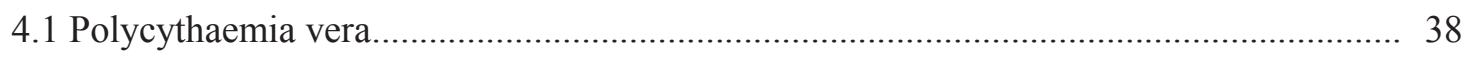

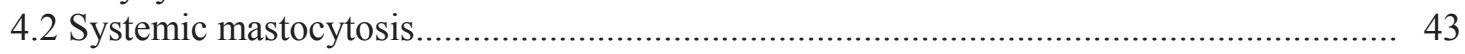

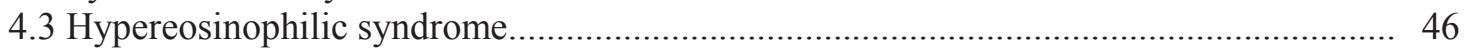

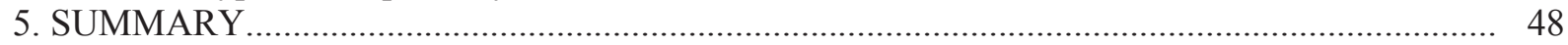

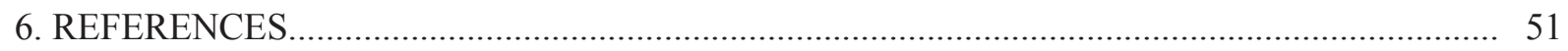

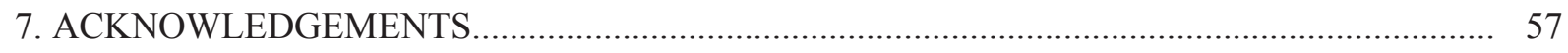

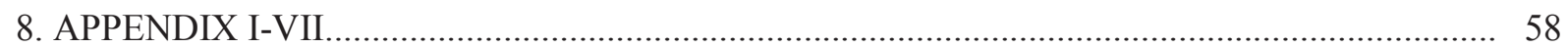




\section{INTRODUCTION}

Myeloproliferative diseases (MPDs) or neoplasms (MPNs) represent a heterogeneous group of clonal haematopoietic stem cell (HSC) disorders characterized phenotypically by an abnormal accumulation of mature-appearing myeloid cells of one or more lineages [1]. The so-called "classic" MPDs - now referred to as "classic" MPNs - include chronic myeloid leukaemia (CML), polycythaemia vera (PV), essential thrombocythaemia (ET), and primary myelofibrosis (PMF) [2]. The Polycythaemia Vera Study Group (PVSG) and the World Health Organization (WHO) classifications distinguish the Philadelphia chromosome (Ph)positive CML from the Ph-negative entities ET, PV, and PMF [3].

Following the discovery of the disease-causing $B C R-A B L 1$ mutation in CML, a number of other fusion genes and oncogenic tyrosine kinase mutations such as Fip1-like-1platelet-derived growth factor receptor alpha gene fusion (FIP1L1-PDGFRA) as well as Kit (KIT D816V) and Janus kinase $2(J A K 2 \mathrm{~V} 617 F)$ mutations have also been identified [4]. The 2008 WHO classification of myeloid neoplasms and acute leukaemia incorporating this new knowledge provides a novel, morphology-, cytogenetics-, and molecular diagnostics-based nomenclature and classification of MPDs [5].

In this revised classification, the expression "myeloproliferative disorder" has been replaced by "myeloproliferative neoplasm". In addition to changes in the nomenclature, a significant modification was - based on their shared features - the enrolment of mast cell diseases (MCDs) among MPNs and the re-organization of chronic eosinophilic leukaemia/hypereosinophilic syndrome (CEL/HES) into "CEL, not otherwise specified (CEL-NOS)" as well as "myeloid and lymphoid neoplasms associated with eosinophilia and abnormalities of PDGFRA, platelet-derived growth factor receptor beta (PDGFRB), or fibroblast growth factor receptor 1 (FGFR1)" (Table 1) [5].

Eight clinicopathological entities fall therefore currently under the category of MPNs: $B C R-A B L 1$-positive $C M L$ and seven $B C R-A B L 1$-negative conditions including chronic neutrophilic leukaemia, PV, PMF, ET, CEL-NOS, mastocytosis, and MPNs - unclassifiable [6]. The term "Ph-negative MPNs" is still widely used as a synonym for classic $B C R-A B L 1$ negative MPNs (PV, ET, PMF) and, in the broader sense, also for other BCRABL1-negative myeloproliferative conditions such as certain rare entities like HES or systemic mastocytosis (SM). 
Table 1. Classification of myeloid neoplasms according to the 2008 WHO scheme [5]

1. Myeloproliferative neoplasms (MPNs)

1.1. Chronic myeloid leukaemia (CML), $B C R-A B L 1$-positive

1.2. Polycythaemia vera (PV)

1.3. Essential thrombocythaemia (ET)

1.4. Primary myelofibrosis (PMF)

1.5. Chronic neutrophilic leukaemia (CNL)

1.6. Chronic eosinophilic leukaemia, not otherwise specified (CEL-NOS)

1.7. Mast cell disease (MCD)

1.8. MPN, unclassifiable

2. Myeloid and lymphoid neoplasms associated with eosinophilia and abnormalities of PDGFRA, PDGFRB, or FGFR1

3. Myelodysplastic/myeloproliferative neoplasms (MDSs/MPNs)

3.1. Chronic myelomonocytic leukaemia (CMML)

3.2. Juvenile myelomonocytic leukaemia (JMML)

3.3. Atypical chronic myeloid leukaemia, $B C R-A B L 1$-negative (aCML)

3.4. MDS/MPN, unclassifiable

4. Myelodysplastic syndromes (MDSs)

5. Acute myeloid leukaemia (AML)

FGFR1, fibroblast growth factor receptor 1; PDGFRA, platelet-derived growth factor receptor alpha; PDGFRB, platelet-derived growth factor receptor beta.

Polycythaemia vera (PV) is the most common entity among all Ph-negative myeloproliferative disorders which, due to its vascular complications, represents an interdisciplinary significance. $\mathrm{PV}$ is characterized by the trilineage clonal proliferation of HSC-derived haematopoietic progenitors resulting in the expansion of the erythrocyte mass. In addition to blood hyperviscosity, the increased red blood cell (RBC) mass results in a higher risk for thrombosis, poor quality of life, and a shorter life expectancy. PV warrants particular attention for the higher risk of cardiovascular $(\mathrm{CV})$ and cerebrovascular events as leading determinants of morbidity and mortality. Predominant are arterial thrombotic events, in particular large vessel arterial events including cerebrovascular events, myocardial infarction, and peripheral arterial occlusion. PV-related haemostatic abnormalities due to qualitative disorders of erythrocytes, neutrophils, and platelets (PLTs) and the pathogenesis of the major thrombotic complications are extensively investigated yet not fully unravelled. From clinical and therapeutical aspects, the role and significance of additional risk factors in the development of PV-associated thrombotic events is of major importance - over the last few years, this topic has been actively investigated.

Systemic mastocytosis (SM), an exceedingly rare of Ph-negative MPNs is considered as an orphan disease with less known clinical presentation, prognosis, and challenging treatment. The multidisciplinary significance of SM can be attributed to the pathological accumulation of morphologically and immunophenotypically abnormal mast cells in one or more organ systems. From mast cell diseases (MCDs) our research focussed on systemic mastocytosis (SM) due to its low incidence rate, heterogeneous manifestation, and clinical complexity and the diagnostic difficulties often associated with it. As the presentation of SM 
may vary from asymptomatic to severe forms, its diagnosis can be especially challenging, both from a clinical and pathological perspective. Up to now, only very limited epidemiological data are available on SM. Although a small set of regional data have been collected through the European Competence Network on Mastocytosis (ECNM), no Hungarian data at all are present.

Among Ph-negative MPNs, hypereosinophilic syndromes (HESs) make up the most heterogeneous and widely debated group of diseases. The clinical presentation of HES is highly variable ranging from a relatively asymptomatic disease to endomyocardial fibrosis. Although HES may also be associated with other organ system failures, cardiac involvement and in particular Loeffler's endocarditis still remain its best known manifestation. As HES represents a very heterogeneous group of diseases, its definition has been strongly debated for decades. Thanks to the current molecular and immunological diagnostic methods, an aetiology-based classification in certain types of HESs is now possible, yet at the price of an even more complicated terminology.

The clinical presentation of HES is highly variable ranging from a relatively asymptomatic disease to endomyocardial fibrosis. A well-known and frequent cardiac manifestation of HES is Loeffler's endocarditis [7-9]. The cardiac involvement begins with eosinophilic infiltration, followed by an intermediate thrombotic stage, and finally evolving into a late fibrotic stage [7]. Enlarged atrium with normal-sized left ventricle (LV) is a minor criterion for endomyocardial fibrosis. At this moment, little is known about left atrial (LA) function in HES. In our research, we investigated a) the ability of a novel non-invasive clinical tool (three-dimensional speckle tracking echocardiography, 3DSTE) to reveal any change in the cardiac functional parameters in a sub-type of HES considered idiopathic in its etiology HES (iHES) without manifest organ damage (as determined by conventional diagnostic methods) as well as b) the left ventricular (LV) rotational mechanics in clinically symptomatic Loeffler's endocarditis.

This work discusses the clinical, laboratory, and molecular characteristics of selected Ph-negative MPNs like PV, SM, and HES and examines how heterogeneous their clinical appearance can be despite their common HSC-derived origin. The diagnosis and treatment of all three Ph-negative MPNs require a strong multidisciplinary (cardiological - neurological dermatological) approach and a close co-operation with other clinical specialists.

\section{AIMS}

The aim of our research was to create a retrospective database of Ph-negative MPNs including three separate cohorts of patients diagnosed with PV, SM, or HES. This work 
evaluates and analyses separately the PV, SM, and HES patient cohorts of the $2^{\text {nd }}$ Department of Internal Medicine and Cardiology Centre, Albert Szent-Györgyi Health Centre, Faculty of Medicine, University of Szeged, with the following objectives in each cohort:

\section{PV cohort:}

Given that PV is the most common type of Ph-negative MPNs and in this group of diseases, vascular events are of outstanding significance and diagnostics, efficacious treatment can only be achieved on a multidisciplinary level, we assessed our patient population in the following aspects:

a) to evaluate the incidence of thrombotic events prior to and during follow-up; and to investigate the main clinical characterteristics of PV patients, either with or without thrombotic complications in the follow-up period;

b) to assess the major cerebrovascular and cardiac thrombotic complications as the most serious thrombotic complications in the PV cohort; and to investigate whether any typical neurological or cardiac lesion(s) could be identified which might be specific to, or characteristic of PV; and

c) to evaluate the contribution of the main $\mathrm{CV}$ risk factors present at time of haematological diagnosis of PV as possible additional risk factors for subsequent thrombotic complications.

\section{SM cohort:}

Analyses in our SM patient group were driven by the orphan nature and the diagnostic, therapeutic, and prognostic difficulties usually associated with this condition as follows:

a) to describe the clinicopathological findings along with bone marrow histological features, molecular characteristics, and laboratory parameters at presentation in a large cohort of SM patients;

b) to evaluate the frequency of KIT D816V mutation in SM subgroups;

c) to estimate the life expectancy of SM patients compared to age- and sex-matched controls; and to evaluate the prognostic relevance of the WHO classification of SM in the investigated patient population; and

d) to quantify the cumulative incidence of SM in the South Great Plain region of Hungary.

\section{HES cohort:}

HES, a condition with highly variable clinical presentation and organ involvement also belongs to the group of rare Ph-negative MPNs. In this patient population the aims of our 
investigations were:

a) to compare LA volumetric, volume-based functional, and strain parameters obtained by three-dimensional speckle-tracking echocardiography (3DSTE), a novel, non-invasive clinical tool for volumetric and strain analysis, between HES patients and matched controls; and

b) to demonstrate LV rotational mechanics in a unique case with Loeffler's endocarditis.

\section{PATIENTS, METHODS, AND RESULTS}

As basis for this research, we retrospectively established a database for scientific research, focussing especially on PV, SM, and HES cases diagnosed at the $2^{\text {nd }}$ Department of Internal Medicine and Cardiology Centre between 1998 and 2014 (Table 2).

Table 2. Overview of the main characteristics of the investigated study population

\begin{tabular}{|l|l|l|l|}
\hline \multirow{2}{*}{\multicolumn{1}{|c|}{ Main characteristics }} & \multicolumn{3}{|c|}{ Patient population } \\
\cline { 2 - 4 } & \multicolumn{1}{|c|}{$\boldsymbol{P V}$} & \multicolumn{1}{c|}{$\boldsymbol{S M}$} & \multicolumn{1}{c|}{ HES } \\
\hline Data collection period & \multicolumn{1}{|c|}{$1998-2014$} & \multicolumn{1}{c|}{$2001-2013$} & $2001-2014$ \\
\hline Total number of patients & $\mathbf{1 0 8}$ & $\mathbf{3 5}$ & $\mathbf{1 1}$ \\
\hline Males $[N(\%)]$ & $57(52.8 \%)$ & $19(54.2 \%)$ & $8(72.7 \%)$ \\
\hline Females $[N(\%)]$ & $51(47.2 \%)$ & $16(45.7 \%)$ & $3(27.2 \%)$ \\
\hline Median age at diagnosis (years) (range) & $62.6(24.8-82.0)$ & $57(31-85)$ & $59(33-77)$ \\
\hline Median follow-up (months) (range) & $54(0.0-16.1)$ & $30.5(1-240)$ & $45(2-168)$ \\
\hline
\end{tabular}

HES, hypereosinophilic syndrome; PV, polycythaemia vera; SM, systemic mastocytosis.

Our investigations were conducted with the approval of the Regional and Institutional Human Medical Biological Research Ethics Committee of the Albert Szent-Györgyi Health Centre, University of Szeged and in accordance with the Declaration of Helsinki principles. Written informed consent was not required from the subjects. The review of trial subjects' relevant medical data was done by using MedSolution, the healthcare data management system of the Albert Szent-Györgyi Health Centre, Faculty of Medicine, University of Szeged.

\subsection{POLYCYTHAEMIA VERA}

\subsubsection{GENERAL CONSIDERATIONS AND BACKGROUND OF THE POLYCYTHAEMIA VERA STUDY}

PV is generally characterized by erythrocytosis, but other signs and symptoms like leukocytosis, thrombocytosis, splenomegaly, vasomotor disturbances, thrombosis, bleeding, 
or pruritus may also be present. The incidence of PV ranges in European Union from 0.4 to 2.8 cases per 100,000 persons per year [10]. PV occurs in all populations and in all age groups including young adults and occasionally children and adolescents, too. The median age at diagnosis was around 61 years (range: 18-95 years) in a group of 1545 patients with WHOdefined PV assessed by the International Working Group for Myeloproliferative Neoplasms Research and Treatment [11]. The incidence of PV was slightly higher in men than in women ( 2.8 vs. 1.3 cases per 100,000 persons per year), with the highest rates in men aged $70-$ 79 years (24 cases per 100,000 persons per year) [12].

The diagnosis of PV is based on the current WHO criteria including clinical and laboratory findings and the molecular analysis of $J A K V 617 F$ mutation [13]. The diagnosis is established if both major criteria and at least one minor criterion, or the first major criterion and at least two minor criteria are present (Table 3).

Table 3. Diagnostic criteria of polycythaemia vera [13]

\section{Major criteria}

1. $\mathrm{Hb}>18.5 \mathrm{~g} / \mathrm{dL}$ in men, $16.5 \mathrm{~g} / \mathrm{dL}$ in women, or other evidence of increased red cell volume

2. Presence of $J A K 2 V 617 F$ or other functionally similar mutation such as $J A K 2$ exon 12 mutation

\section{Minor criteria}

1. Bone marrow biopsy showing hypercellularity for age with trilineage growth (panmyelosis) with prominent erythroid, granulocytic, and megakaryocytic proliferation

2. Serum erythropoietin level below the reference range for normal

3. Endogenous erythroid colony formation in vitro

$\mathrm{Hb}$, haemoglobin; JAK2, Janus kinase 2.

$J A K 2 V 617 F$ is by far the most prevalent mutation in $B C R-A B L 1$-negative MPNs: it occurs in $\sim 95 \%$ of patients with PV, in $\sim 55 \%$ with ET, and in $\sim 65 \%$ with PMF, respectively [6].

The Janus kinase/signal transducer and activator of transcription (JAK-STAT) signalling pathway is of central importance in a number of cellular processes including proliferation, survival, and normal functioning of haematopoietic and other cells [6]. The vast majority ( $~ 96 \%)$ of PV patients have a JAK2 V617F somatic activating mutation in exon 14 while the rest of them ( $3 \%)$ exhibit a $J A K 2$ exon 12 mutation [14]. The overall median survival in PV is 14 years with a median survival time of 24 years for patients younger than 60 years [15]. The 10 -year risk is $3 \%$ and $10 \%$ for leukaemic transformation and fibrotic transformation, respectively [16]. Leukaemic transformation rates at 20 years are estimated at $<10 \%$ in PV [17].

In contrast, the risk of thrombosis in PV is high: the prevalence of major thrombotic events (arterial events: acute myocardial infarction [AMI], ischaemic stroke, transient ischaemic attack [TIA]; venous events: deep vein thrombosis [DVT], pulmonary embolism [PE], splanchnic thrombosis) at the time of diagnosis ranges approximately $34 \%$ to $39 \%$; 
corresponding values for thrombosis at follow-up are approximately $8 \%$ to $19 \%$. Concerning major thrombotic events, arterial complications occur more often than venous ones. Clinical manifestation of these thrombotic events is mainly discussed only in isolated case reports or case series. A large proportion of patients suffer from vasomotor disturbances (e.g. headache, dizziness, erythromelalgia, acral paraesthesias, atypical chest pain) or pruritus [18]. Some patients may also develop acquired von Willebrand syndrome, especially those with extreme thrombocytosis $\left(\mathrm{PLT}>1,000 \times 10^{9} / \mathrm{L}\right)$ in both PV and ET and are at risk for acetylsalicylic acid (ASA)-associated bleeding [17]. Thrombosis is a leading cause of morbidity and mortality in $\mathrm{PV}$. The pathogenesis of acquired thrombophilic state in PV is multifactorial and complex. Currently, two main mechanisms are considered as of crucial role: on the one hand, the abnormalities of blood cells (platelets, RBCs, and white blood cells [WBCs]) arising from the clonal proliferation of haematopoietic progenitor cells and the acquisition of a prothrombotic phenotype and on the other hand, the host inflammatory response to cytokines and other mediators secreted by the malignant cells as well as the procoagulant activity of vascular cells triggered by these proinflammatory stimuli [19]. Abnormalities of the clonal proliferation of HSCs include not only quantitative changes but also qualitative modifications that characterize the switch of these cells from a resting to a procoagulant phenotype [20].

\subsubsection{STUDY POPULATION AND DATA COLLECTION}

To establish our PV database, we relied on two sources. First, we screened medical records (MedSolution) of patients presented at the Haematology Outpatient Unit, $2^{\text {nd }}$ Department of Internal Medicine and Cardiology Centre with the orienting diagnosis of different chronic MPDs, per appropriate ICD (International Classification of Diseases, ICD-10) codes and in a given time period; and second, we checked data of patients subject to molecular diagnostic testing for myeloproliferative disorders at the Department of Medical Genetics, Faculty of Medicine, University of Szeged. Finally, we set up an integrated database including the relevant elements of both data sources.

For our research, medical records of a total of 933 patients were screened. Out of them, the definite diagnosis of PV fulfilling WHO criteria could be established in 108 cases. Data of these 108 subjects made up our "PV Database 1998-2014" (Table 4). 
Table 4. Main demographic and clinicohaematological characteristics of the polycythaemia vera population

\begin{tabular}{|l|l|}
\hline \multicolumn{1}{|c|}{ Main characteristics of the PV cohorts } & \multicolumn{1}{|c|}{ Database 1998-2014 } \\
\hline Total number of patients & $\mathbf{1 0 8}$ \\
\hline Males [N, (\%)] & $57(52.8 \%)$ \\
\hline Females [N, (\%)] & $51(47.2 \%)$ \\
\hline Age at diagnosis, median (years) (range) & $62.6(24.8-82.0)$ \\
\hline Median follow-up (months) (range) & $54(0.0-16.1)$ \\
\hline Median Hb (g/L) & $174.1 \pm 24.0$ \\
\hline Median leukocyte count at diagnosis (range) $(\mathrm{G} / \mathrm{L})$ & $11.2 \pm 4.6(5.2-31.8)$ \\
\hline Median platelet count at diagnosis (range) $(\mathrm{G} / \mathrm{L})$ & $398.8 \pm 232.7(65-1329)$ \\
\hline JAK2 V617F-positive cases [N, (\%)] & $102(94.4 \%)$ \\
\hline Conventional risk factors in PV & \\
\hline Age $>60$ years [N, (\%)] & $62(57.4 \%)$ \\
\hline Prior thrombotic events & $33(30.5 \%)$ \\
\hline Low risk [N, (\%)] & $36(33.3 \%)$ \\
\hline High risk [N, (\%)] & $72(66.7 \%)$ \\
\hline
\end{tabular}

$\mathrm{Hb}$, haemoglobin; JAK2, Janus kinase 2; PV, polycythaemia vera.

Thrombotic events prior to and following the clinical diagnosis were retrospectively collected for each PV patient, with special focus on CV (AMI), cerebrovascular (stroke, TIA, vertebrobasilar insufficiency [VBI]), and venous thrombotic events (DVT, PE, splanchnic vein thrombosis).

Data on $\mathrm{CV}$ risk factors present at time of PV clinical diagnosis including hypertension, tobacco use, diabetes mellitus (DM), hyperlipidaemia (hypercholesterolaemia or hypertriglyceridaemia or both), and obesity (body mass index $>30 \mathrm{~kg} / \mathrm{m}^{2}$ ) were also collected (Table 5).

Table 5. Distribution of cardiovascular risk factors at time of the clinical diagnosis of polycythaemia vera

\begin{tabular}{|l|l|}
\hline Distribution of CV risk factors in PV patients & [N (\%)] \\
\hline Hypertension & $73(67.6 \%)$ \\
\hline Hyperlipidaemia & $32(29.6 \%)$ \\
\hline Diabetes mellitus & $23(21.3 \%)$ \\
\hline Tobacco use & $21(19.4 \%)$ \\
\hline Obesity $\left(\mathrm{BMI}>30 \mathrm{~kg} / \mathrm{m}^{2}\right)$ & $30(27.8 \%)$ \\
\hline
\end{tabular}

$\mathrm{BMI}$, body mass index; $\mathrm{CV}$, cardiovascular; $\mathrm{PV}$, polycythaemia vera.

In the haematological management of PV patients, a risk-oriented strategy was adopted: selected low-risk patients received anti-platelet therapy while cytoreductive drugs (e.g. hydroxyurea) in combination with anti-platelet medication were administered to highrisk patients. Phlebotomy was reserved for low-risk patients and for those at high risk before 
9

cytoreductive treatment in order to reach the recommended target haematocrit (Hct) value of $<0.45$ (Table 6) [21].

Table 6. Haematological treatment of polycythaemia vera patients

\begin{tabular}{|l|l|}
\hline \multicolumn{1}{|c|}{ Haematological treatment of PV patients } & \multicolumn{1}{c|}{$[\mathbf{N}(\%)]$} \\
\hline Phlebotomy & $51(47.2 \%)$ \\
\hline Platelet aggregation inhibitor (ASA) & $79(73.1 \%)$ \\
\hline ASA + phlebotomy & $41(38.0 \%)$ \\
\hline Cytoreductive treatment (hydroxyurea) & $50(46.3 \%)$ \\
\hline
\end{tabular}

ASA, acetylsalicylic acid; PV, polycythaemia vera.

\subsubsection{STATISTICAL ANALYSES}

Clinical data were collected using Microsoft ${ }^{\circledR}$ Excel $^{\circledR} 2010$ software and subjected to statistical analysis with STATISTICA v9.1 (StatSoft, Hungary) and SPSS 20 (IBM, USA) softwares.

\section{Investigation of the contribution of cardiovascular risk factors in polycythaemia vera}

To evaluate and compare the overall effect of $\mathrm{CV}$ risk factors present at time of haematological diagnosis, Mann-Whitney $U$ tests were performed both for the presence and the absence of thrombotic complications, i.e., CV (AMI), cerebrovascular (ischaemic stroke, TIA, VBI), and venous thrombotic events (DVT, PE, splanchnic vein thrombosis). In addition to each predefined CV risk factor (hypertension, hyperlipidaemia, tobacco use, DM, and obesity), the effect of only one CV risk factor, the effect of two or more CV risk factors as well as the role of leukocytosis $(>11.1 \mathrm{G} / \mathrm{L})$ or increased Hct $(>45 \%)$ were also investigated. Statistical significance was set at $5 \%$ and, as reasoned by study population size, also considered at $10 \%$.

To evaluate and compare the probability of thrombosis-free survival for PV patients a) without $\mathrm{CV}$ risk factors and with at least one $\mathrm{CV}$ risk factor and b) with at most one CV risk factor and with two or more CV risk factors, the Kaplan-Meier method was used, combined with log-rank (Mantel-Cox) tests [22].

\subsubsection{LABORATORY METHODS}

Samples for $J A K 2 V 617 F$ molecular analyses were obtained from the DNA bank of the Department of Medical Genetics based on the information on PV patients diagnosed at the $2^{\text {nd }}$ Department of Internal Medicine and Cardiology Centre between 1998 and 2014. DNA was isolated from ethylenediaminetetraacetic acid (EDTA) -stabilized peripheral blood samples and screened for $J A K 2$ V617F mutation using allele-specific polymerase chain reaction (PCR) method as part of the routine diagnostic protocol [23]. For patients whose 
haematological diagnosis was established before $J A K 2$ V617F mutation screening had become an obligatory part of the diagnostic protocol, samples for genetic testing were collected and analyzed retrospectively.

Serum erythropoietin levels were determined by chemiluminescent immunoassay (Siemens Immulite) at the Laboratory of Endocrinology, University of Szeged [24].

\subsubsection{RESULTS}

THROMBOTIC EVENTS PRIOR TO AND DURING FOLLOW-UP AND THE MAIN CLINICAL CHARACTERTERISTICS OF POLYCYTHAEMIA VERA PATIENTS WITH OR WITHOUT THROMBOTIC COMPLICATIONS IN THE FOLLOW-UP PERIOD (Aim I.a)

The retrospective analysis of all recorded events revealed altogether 33 pre-diagnosis vascular events in 108 (30.5\%) patients: 17 cerebrovascular events (stroke/TIA), $8 \mathrm{CV}$ events (AMI), and 8 venous thrombotic events. During the haematological follow-up after the diagnosis of PV, a total of 20 events were observed in 108 (18,5\%) patients: 11 cerebrovascular events (stroke/TIA), $7 \mathrm{CV}$ events (AMI), and 2 venous thrombotic events (Table 7).

Table 7. Vascular events before the clinical diagnosis of polycythaemia vera and during follow-up

\begin{tabular}{|l|l|}
\hline Type of the vascular event & Number of the vascular events \\
\hline Prediagnostic major vascular events & $\mathbf{3 3}$ \\
\hline Cerebrovascular events (stroke/TIA/VBI) & 17 \\
\hline CV events (AMI) & 8 \\
\hline Venous thrombotic events & 8 \\
\hline Follow-up & $\mathbf{2 0}$ \\
\hline Cerebrovascular events (stroke/TIA/VBI) & 11 \\
\hline CV events (AMI) & 7 \\
\hline Venous thrombotic events & 2 \\
\hline
\end{tabular}

TIA, transient ischaemic attack; VBI, vertebrobasilar insufficiency; CV, cardiovascular; AMI, acute myocardial infarction.

We investigated whether subjects with or without a post-diagnostic history of thrombotic events significantly differ in their main clinical characteristics (Table 8).

Mann-Whitney tests were performed in the cases of the presence or absence of thrombotic events after the diagnosis of PV, comparing the overall effects of series variables: mean follow-up, mean age at diagnosis, JAK2 V617F-positivity, haematology blood test results, organomegaly, number of thrombotic events before heamatological diagnosis, vascular risk factors, and treatment. Fisher's exact test results were considered for sex and conventional risk groups. Some CV risk factors as hypertension $(\mathrm{p}=0.001)$ and tobacco use ( $p=0.023$ ) were significantly different in our two patient groups; the different thrombotic risk of conventional low- and high-risk groups $(\mathrm{p}=0.029)$ was confirmed as well. 
11

Table 8. Characteristics of polycythaemia vera patients with or without thrombotic complications during follow-up

\begin{tabular}{|c|c|c|c|}
\hline MAIN CLINICAL CHARACTERISTICS & $\begin{array}{l}\text { PATIENTS WITH } \\
\text { THROMBOTIC } \\
\text { COMPLICATIONS }\end{array}$ & $\begin{array}{l}\text { PATIENTS WITHOUT } \\
\text { THROMBOTIC } \\
\text { COMPLICATIONS }\end{array}$ & P-VALUE \\
\hline Number of patients $N=108(100 \%)$ & 18 & 90 & \\
\hline Male $[\mathrm{N}(\%)]$ & $12(66.7 \%)$ & $45(50.0 \%)$ & \multirow{2}{*}{0.198} \\
\hline Female $[\mathrm{N}(\%)]$ & $6(33.3 \%)$ & $45(50.0 \%)$ & \\
\hline Mean follow-up (years) & $3.9(0.1-11.1)$ & $5.8(0.0-23.0)$ & 0.273 \\
\hline Mean age at diagnosis (years) & $64.9(38.2-78.5)$ & $59.8(24.8-82.0)$ & 0.106 \\
\hline$J A K 2$ V617F-positivity [N (\%)] & $16(88.9 \%)$ & $86(95.6 \%)$ & 0.256 \\
\hline \multicolumn{4}{|c|}{ Haematology blood test results at time of haematological diagnosis } \\
\hline Mean platelet count $(\mathrm{G} / \mathrm{L})$ & $467.7 \pm 274.8$ & $\beta 85.0 \pm 222.5$ & 0.255 \\
\hline Mean white blood cell count (G/L) & $12.7 \pm 6.1$ & $10.9 \pm 4.3$ & 0.463 \\
\hline Mean red blood cell count $(\mathrm{T} / \mathrm{L})$ & $6.4 \pm 0.8$ & $6.1 \pm 1.2$ & 0.059 \\
\hline Mean haemoglobin $(\mathrm{g} / \mathrm{L})$ & $178.7 \pm 27.4$ & $173.1 \pm 23.3$ & 0.316 \\
\hline \multicolumn{4}{|c|}{ Organomegaly at time of haematological diagnosis } \\
\hline Hepatomegaly & $6(33.3 \%)$ & $33(36.7 \%)$ & 0.789 \\
\hline Splenomegaly & $6(33.3 \%)$ & $26(28.9 \%)$ & 0.707 \\
\hline Hepatosplenomegaly & $2(11.1 \%)$ & $19(21.1 \%)$ & 0.330 \\
\hline \multicolumn{4}{|l|}{ Number of thrombotic events } \\
\hline Before heamatological diagnosis & 6 & 27 & 0.937 \\
\hline After heamatological diagnosis & 20 & 0 & - \\
\hline \multicolumn{4}{|l|}{ Vascular risk factors } \\
\hline Hypertension & $18(100.0 \%)$ & $55(61.1 \%)$ & 0.001 \\
\hline Tobacco use & $0(0.0 \%)$ & $21(23.3 \%)$ & 0.023 \\
\hline Diabetes mellitus & $5(27.8 \%)$ & $18(20.0 \%)$ & 0.464 \\
\hline Obesity & $8(44.4 \%)$ & $22(24.4 \%)$ & 0.085 \\
\hline Hyperlipidaemia & $8(44.4 \%)$ & $24(26.7 \%)$ & 0.133 \\
\hline \multicolumn{4}{|c|}{ Patient distribution by conventional risk categories } \\
\hline Low-risk group & $2(11.1 \%)$ & $34(37.8 \%)$ & \multirow{2}{*}{0.029} \\
\hline High-risk group & $16(88.9 \%)$ & $56(62.2 \%)$ & \\
\hline \multicolumn{4}{|l|}{ Treatment } \\
\hline Hydroxyurea & $10(55.6 \%)$ & $40(44.4 \%)$ & 0.390 \\
\hline Acetylsalicylic acid & $14(77.8 \%)$ & $65(72.2 \%)$ & 0.629 \\
\hline Phlebotomy & $10(55.6 \%)$ & $41(45.6 \%)$ & 0.440 \\
\hline
\end{tabular}




\section{CARDIOVASCULAR AND CEREBROVASCULAR COMPLICATIONS (Aim I.b)}

\section{Cardiovascular complications}

Detailed clinical data and coronary angiography findings for adequate cardiological analyses of $\mathrm{CV}$ complications in PV patients during follow-up were available for 6 patients (1 male, 5 females; mean age 69.5 years [range: $64-76$ years]).

Mean Hct was $52.8 \pm 8.6 \%$ at time of haematological diagnosis and $49.5 \pm 7.9 \%$ at the onset of AMI. Median WBC count was $11.28 \pm 5.7 \mathrm{G} / \mathrm{L}$ at haematological diagnosis while by the onset of AMI, it increased to a level of $13.47 \pm 5.8 \mathrm{G} / \mathrm{L}$.

Five (83.3\%) out of the six analyzed patients exhibited JAK2 V617F mutation. Most of the patients $(83.3 \%)$ had at least two major conventional CV risk factors.

Non-ST-segment elevation myocardial infarction (NSTEMI) was diagnosed in all six PV patients by coronary angiography. Significant stenosis of coronary arteries requiring percutaneous coronary intervention with stent implantation was seen in two patients. Coronary angiography showed only normal epicardial coronary arteries, non-significant stenosis, or distal occlusion in one patient each. One patient underwent coronary artery bypass grafting and saphenous vein graft stenting. Evaluated cases are listed individually in Table 9. 
Table 9. Presentation of individual polycythaemia vera patients with cardiovascular complications enrolled in the detailed analyses

\begin{tabular}{|c|c|c|c|c|c|c|}
\hline \multirow{2}{*}{$\begin{array}{l}\text { CASE No. } \\
\text { AGE/SEX/DA } \\
\text { TE OF } \\
\text { DIAGNOSIS }\end{array}$} & \multirow{2}{*}{$\begin{array}{c}\text { TIME } \\
\text { BETWEEN } \\
\text { CARDIOLOGIC } \\
\text { AL EVENT AND } \\
\text { PV } \\
\text { DIAGNOSIS } \\
\end{array}$} & \multirow{2}{*}{$\begin{array}{l}\text { CV RISK } \\
\text { FACTORS } \\
\text { PRESENT AT PV } \\
\text { DIAGNOSIS }\end{array}$} & \multirow{2}{*}{$\begin{array}{c}J A K 2 \text { V617F } \\
\text { MUTATION }\end{array}$} & \multicolumn{2}{|c|}{ CARDIOLOGICAL COMPLICATIONS } & \multirow{2}{*}{$\begin{array}{l}\text { HAEMATOLOGICAL } \\
\text { TREATMENT AFTER PV } \\
\text { DIAGNOSIS }\end{array}$} \\
\hline & & & & $\begin{array}{l}\text { CARDIOLOGICAL } \\
\text { PRESENTATION }\end{array}$ & CORONARY ANGIOGRAPHY FINDINGS & \\
\hline $\begin{array}{c}\text { CASE 1 } \\
72 / M / 2005\end{array}$ & 8 months & $\begin{array}{l}\text { hypertension, } \\
\text { hyperlipidaemia, } \\
\text { obesity }\end{array}$ & negative & NSTEMI & $\begin{array}{l}\text { LAD: diagonal borderline lesion } \\
\text { LCX: first OM branch } 20 \% \text { stenosis } \\
\text { RC: ostial } 80 \% \text { stenosis (PCI + stent implantation) }\end{array}$ & $\begin{array}{l}\text { ASA + clopidogrel }+ \\
\text { phlebotomy }\end{array}$ \\
\hline $\begin{array}{c}C A S E 2 \\
63 / F / 2010\end{array}$ & 15 months & hypertension & positive & NSTEMI & $\begin{array}{l}\text { LAD: proximal } 90 \% \text { stenosis }(\mathrm{PCI}+\text { stent implantation) } \\
\text { LCX: normal } \\
\text { RC: } 50 \% \text { stent stenosis }\end{array}$ & ASA + clopidogrel \\
\hline $\begin{array}{c}\text { CASE 3 } \\
74 / F / 2005\end{array}$ & 41 months & $\begin{array}{c}\text { hypertension, } \\
\text { hyperlipidaemia }\end{array}$ & positive & NSTEMI & $\begin{array}{l}\text { LAD: proximal } 40 \% \text { stenosis } \\
\text { LCX: normal } \\
\text { RC: normal }\end{array}$ & $\begin{array}{l}\text { Hydroxyurea }+ \\
\text { phlebotomy }\end{array}$ \\
\hline $\begin{array}{c}\text { CASE 4 } \\
76 / F / 2009\end{array}$ & 13 months & $\begin{array}{l}\text { hypertension, } \\
\text { hyperlipidaemia, } \\
\text { obesity, DM }\end{array}$ & positive & NSTEMI & $\begin{array}{l}\text { LAD: ostial occlusion, LIMA-LAD (normal) } \\
\text { LCX: } 95 \% \text { stenosis, proximal } 70 \% \text { stenosis of SVG-LCX } \\
\text { (stent in SVG) } \\
\text { RC: proximal occlusion } \\
\text { (CABG) }\end{array}$ & ASA + phlebotomy \\
\hline $\begin{array}{c}\text { CASE } 5 \\
64 / F / 2013\end{array}$ & 8 months & $\begin{array}{l}\text { hypertension, } \\
\text { obesity }\end{array}$ & positive & NSTEMI & $\begin{array}{l}\text { LAD: normal } \\
\text { LCX: normal } \\
\text { RC: normal }\end{array}$ & ASA \\
\hline $\begin{array}{c}\text { CASE } 6 \\
68 / F / 2011\end{array}$ & 4 months & $\begin{array}{l}\text { hypertension, } \\
\text { obesity, DM }\end{array}$ & positive & NSTEMI & $\begin{array}{l}\text { LAD: normal } \\
\text { LCX: normal } \\
\text { RC: distal occlusion }\end{array}$ & ASA + hydroxyurea \\
\hline
\end{tabular}

ASA, acetylsalicylic acid; CABG, coronary artery bypass grafting; CV, cardiovascular; DM, diabetes mellitus; F, female; LAD, left anterior descending coronary artery; LCX, left circumflex coronary artery; LIMA, left internal mammary artery; LM, left main coronary artery; M, male; NSTEMI, non-ST-segment elevation myocardial infarction; OM, obtuse marginal artery; PCI, percutaneous coronary intervention; PV, polycythaemia vera; RC, right coronary artery; SVG, saphenous vein graft. 


\section{Cerebrovascular complications}

Detailed analysis of cerebrovascular events was performed in 11 out of the $108 \mathrm{PV}$ patients enrolled (9 males, 2 females; median age: 65 years [range: 52-79 years]). JAK2 V617F mutation positivity was detected in 10 of the 11 analyzed cases (91\%). Most of the patients $(8 / 11,72 \%)$ had at least two major conventional vascular risk factors (e.g. hypertension, hyperlipidaemia, DM, or obesity).

Mean Hct level at onset of cerebrovascular complications after the initiation of specific haematological treatment was lower (45\% [range: 41-57\%]) than at PV haematological diagnosis (54\% [range: 45-66\%]). Mean WBC at time of haematological diagnosis was $13 \mathrm{G} / \mathrm{L}$ (range: 5-24 G/L) and persisted (13 G/L [range: 5-25 G/L]) during the course of post-treatment cerebrovascular thrombotic events.

In most of the cases (7/11 patients, $63 \%)$, chronic ischaemic white matter lesions were detected on brain computed tomography scan obtained at cerebrovascular event onset. In addition, mild cerebral atrophy was also a frequent finding. The clinical presentation was predominated by lacunar syndromes or VBI. Two patients - one of them on anticoagulant therapy - sustained haemorrhagic stroke. Overall, these data allow us to suppose that after adjusting for major conventional vascular risk factors, PV predisposes to small vessel cerebral disease manifested mainly as lacunar syndromes, even if most of the patients had additional vascular risk factors, too. Evaluated cases are listed individually in Table 10. 
Table 10. Main characteristics of polycythaemia vera patients with cerebrovascular event

\begin{tabular}{|c|c|c|c|c|c|}
\hline \multirow{2}{*}{$\begin{array}{c}\text { CASE No. } \\
\text { AGE/SEX/DATE } \\
\text { OF DIAGNOSIS }\end{array}$} & \multirow[b]{2}{*}{ CV RISK FACTORS } & \multicolumn{2}{|c|}{ CEREBROVASCULAR COMPLICATIONS } & \multicolumn{2}{|c|}{ TREATMENT } \\
\hline & & $\begin{array}{l}\text { NEUROLOGICAL } \\
\text { PRESENTATION }\end{array}$ & CT/MRI FINDINGS & AFTER PV DIAGNOSIS & $\begin{array}{l}\text { AFTER THE FIRST CEREBROVASCULAR } \\
\text { EVENT }\end{array}$ \\
\hline $\begin{array}{c}\text { CASE 1 } \\
72 / \mathrm{M} / 2005\end{array}$ & $\begin{array}{c}\text { hypertension, } \\
\text { hyperlipidaemia, obesity }\end{array}$ & 2011: VBI & $\begin{array}{l}\text { CT: mild cerebral atrophy } \\
\text { medium-sized chronic ischaemic white matter } \\
\text { lesions }\end{array}$ & ASA + phlebotomy & clopidogrel + phlebotomy \\
\hline $\begin{array}{c}C A S E 2 \\
64 / M / 2010\end{array}$ & $\begin{array}{l}\text { hypertension, } \\
\text { hyperlipidaemia, } \\
\text { DM }\end{array}$ & 2011: VBI & $\begin{array}{l}\text { CT: no pathological lesion } \\
\text { MRI (2005): mild chronic ischaemic white matter } \\
\text { lesions }\end{array}$ & ASA + phlebotomy & clopidogrel + hydroxyurea \\
\hline $\begin{array}{c}\text { CASE } 3 \\
70 / \mathbf{M} / 2008\end{array}$ & $\begin{array}{c}\text { hypertension, } \\
\text { hyperlipidaemia, } \\
\text { MTHFR C677T } \\
\text { homozygous } \\
\text { polymorphism (with } \\
\text { currently normal } \\
\text { homocystein levels) } \\
\end{array}$ & $\begin{array}{l}\text { 05/2011: left MCA ischaemic } \\
\text { stroke (mild), dementia (mixed) } \\
\text { 11/2011: left hemispheric } \\
\text { haemorrhagic stroke }\end{array}$ & $\begin{array}{l}\text { CT }(05 / 2011) \text { : mild cerebral atrophy, lacunes in } \\
\text { left basal ganglia, mild chronic left-sided } \\
\text { ischaemic white matter lesions } \\
\text { CT }(11 / 2011) \text { : acute parenchymal haemorrhage } \\
(3 \times 6 \mathrm{~cm}) \text { in the left parieto-temporal region } \\
\text { (concomitant anticoagulant therapy for AF); left } \\
\text { MCA stenosis }\end{array}$ & $\mathrm{ASA}+$ phlebotomy $+($ warfarin for $\mathrm{AF})$ & $\begin{array}{l}\text { ASA + hydroxyurea }+ \text { anticoagulant } \\
\text { treatment (with low-therapeutic INR) }\end{array}$ \\
\hline $\begin{array}{c}\text { CASE } 4 \\
79 / \mathrm{M} / 2001\end{array}$ & hypertension & 2008: vertigo, suspected VBI & $\begin{array}{l}\text { CT: mild cerebral atrophy, some lacunes in basal } \\
\text { ganglia, mild chronic ischaemic white matter } \\
\text { lesions }\end{array}$ & $\mathrm{ASA}+$ phlebotomy + hydroxyurea & ASA + phlebotomy + hydroxyurea \\
\hline $\begin{array}{c}\text { CASE 5 } \\
53 / \mathrm{M} / 2003\end{array}$ & $\begin{array}{c}\text { hypertension, } \\
\text { hyperlipidaemia, } \\
\text { obesity }\end{array}$ & $\begin{array}{l}\text { 2007: VBI } \\
\text { 2010: VBI }\end{array}$ & $\begin{array}{l}\text { CT: not available } \\
\text { CT: mild cerebral atrophy }\end{array}$ & ASA + phlebotomy & $\begin{array}{l}\mathrm{ASA}+\text { phlebotomy }+ \text { hydroxyurea }+ \\
\text { pentoxifylline }\end{array}$ \\
\hline $\begin{array}{c}\text { CASE 6 } \\
65 / \mathrm{M} / 2011\end{array}$ & hypertension, DM & 2013: right MCA stroke (mild) & $\begin{array}{l}\text { CT: acute infarction }(2 \times 2 \mathrm{~cm}) \text { in right } \\
\text { hemispheric white matter }\end{array}$ & phlebotomy & (warfarin for $A F$ ) \\
\hline $\begin{array}{c}C A S E 7 \\
76 / M / 2006\end{array}$ & $\begin{array}{l}\text { hypertension, } \\
\text { obesity }\end{array}$ & 2012: left MCA stroke (mild) & $\begin{array}{l}\text { CT: medium-degree cerebral atrophy, chronic } \\
\text { periventricular white matter lesions }\end{array}$ & $\begin{array}{c}\text { ASA +hydroxyurea + (acenocoumarol } \\
\text { for } A F)\end{array}$ & clopidogrel + (acenocoumarol for AF) \\
\hline $\begin{array}{c}\text { CASE 8 } \\
57 / \mathrm{M} / 2008\end{array}$ & $\begin{array}{c}\text { hypertension, } \\
\text { hyperlipidaemia, DM, } \\
\text { obesity }\end{array}$ & $\begin{array}{l}\text { 2008: right MCA stroke (mild) } \\
\text { 2010: worsening of chronic } \\
\text { neurological signs (dysarthria) }\end{array}$ & $\begin{array}{l}\text { CT }(2008,2010) \text { : cerebral atrophy, lacunes in } \\
\text { basal ganglia, extensive chronic ischaemic white } \\
\text { matter lesions }\end{array}$ & ASA & clopidogrel \\
\hline $\begin{array}{c}C A S E 9 \\
52 / F / 1998\end{array}$ & $\begin{array}{l}\text { hypertension, } \\
\text { PAD }\end{array}$ & 2004: TIA (VBI) & CT: negative & ASA + hydroxyurea (acenocoumarol) & $\begin{array}{c}\text { hydroxyurea + clopidogrel + } \\
\text { (acenocoumarol) }\end{array}$ \\
\hline $\begin{array}{c}C A S E 10 \\
77 / M / 2012 \\
\end{array}$ & hypertension & $\begin{array}{l}\text { 2012: fatal right MCA } \\
\text { haemorrhagic stroke }\end{array}$ & $\begin{array}{l}\text { CT: right-sided space-occupying haemorrhage in } \\
\text { basal ganglia with intraventricular extension, } \\
\text { chronic white matter lesions }\end{array}$ & ASA & \\
\hline $\begin{array}{c}C A S E 11 \\
54 / \text { F/2013 }\end{array}$ & $\begin{array}{l}\text { hypertension, } \\
\text { hyperlipidaemia, } \\
\text { obesity }\end{array}$ & 2014: vertigo - VBI & CT: not available & ASA & clopidogrel \\
\hline
\end{tabular}

AF, atrial fibrillation; ASA, acetylsalicylic acid; CT, computed tomography; CV, cardiovascular; DM, diabetes mellitus; F, female; M, male; MCA, middle cerebral artery; MRI, magnetic resonance imaging; PAD, peripheral arterial disease; PCA, posterior cerebral artery; PV, polycythaemia vera; TIA, transient ischaemic attack; VBI, vertebrobasilar insufficiency. 
THE CONTRIBUTION OF CARDIOVASCULAR RISK FACTORS TO SUBSEQUENT THROMBOTIC COMPLICATIONS $($ Aim I.c)

Univariate analyses revealed a significant overall association between thrombotic complications and high blood pressure $(p=0.000)$, tobacco use $(p=0.014)$, and obesity $(p=0.078)$. Hyperlipidaemia $(p=0.112)$ and $\mathrm{DM}(\mathrm{p}=0.323)$ were not associated with an increased risk of subsequent thrombosis. The presence of one CV risk factor $(p=0.016)$ or two or more $\mathrm{CV}$ risk factors $(\mathrm{p}=0.024)$ significantly increased the occurrence of thrombotic complications. Leukocytosis ( $\mathrm{WBC}>11.1 \mathrm{G} / \mathrm{L}$ ), however, did not increase significantly the risk of thrombotic events $(\mathrm{p}=0.119)$. The frequency of thrombotic events during follow-up differed significantly between PV subgroups with Hct values below or above $45 \%(p=0.089)$ (Table 11).

Table 11. Mann-Whitney U test results in subgroups of polycythaemia vera patients sustaining or not sustaining thrombotic events during follow-up

\begin{tabular}{|c|c|}
\hline \multicolumn{2}{|c|}{ Comparison of PV patients who did or did not sustain thrombotic events during follow-up } \\
\hline VARIABLES & $\begin{array}{l}\text { MANN-WHITNEY UNIVARIATE ANALYSIS p- } \\
\text { VALUE }\end{array}$ \\
\hline \multicolumn{2}{|l|}{ CV risk factors } \\
\hline Hypertension & $0.000 * *$ \\
\hline Hyperlipidaemia & 0.112 \\
\hline Tobacco use & $0.014 * *$ \\
\hline Diabetes mellitus & 0.323 \\
\hline Obesity & $0.078^{*}$ \\
\hline Presence of 0 or $1 \mathrm{CV}$ risk factor & $0.016 * *$ \\
\hline Presence of $\geq 2 \mathrm{CV}$ risk factors & $0.024 * *$ \\
\hline White blood cell count $>11.1 \mathrm{G} / \mathrm{L}$ & 0.119 \\
\hline Haematocrit $>45 \%$ & $0.089 *$ \\
\hline
\end{tabular}

Significant differences at $10 \%$ are marked by $*$ and those at $5 \%$ by $* *$.

$C V$, cardiovascular; $P V$, polycythaemia vera.

To compare the thrombosis-free survival of patients in the presence or absence of the investigated CV risk factors, Kaplan-Meier curves and log-rank tests (Mantel-Cox) were used which indicated a significant difference of the thrombosis-free survival between PV patients without $\mathrm{CV}$ risk factors $(\mathrm{N}=20)$ and those with at least one $\mathrm{CV}$ risk factor $(\mathrm{N}=88)$ $(\mathrm{p}=0.017)$ (Figure 1). 


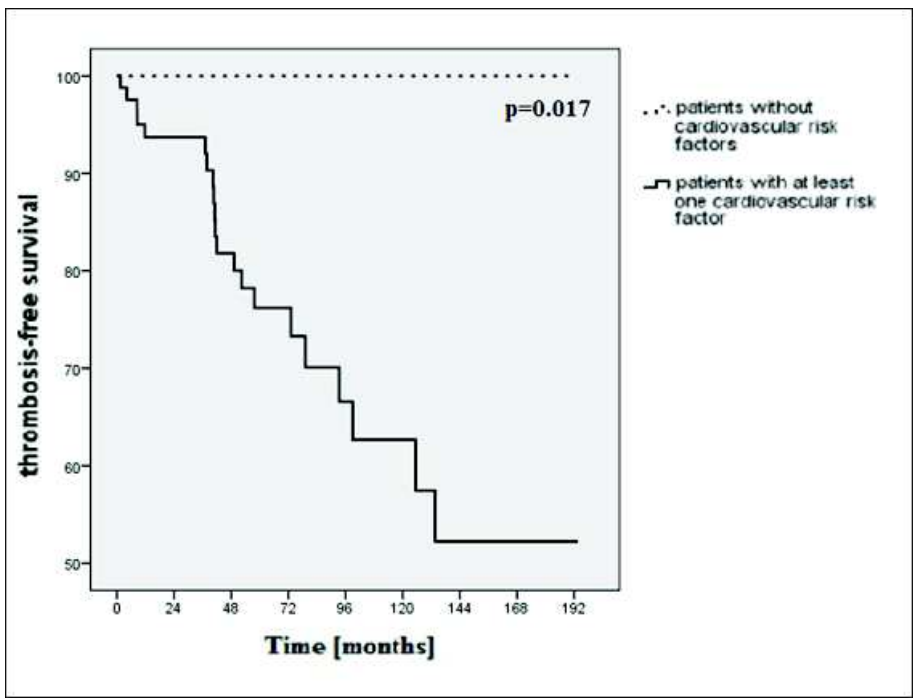

Figure 1. Probability of thrombosis-free survival during haematological follow-up in subgroups of polycythaemia vera patients without cardiovascular risk factors and with at least one cardiovascular risk factor

A significant difference was also observed between PV patients with at most one CV risk factor $(\mathrm{N}=49)$ and $\mathrm{PV}$ patients with two or more $\mathrm{CV}$ risk factors $(\mathrm{N}=59)(\mathrm{p}=0.011)$ (Figure 2).

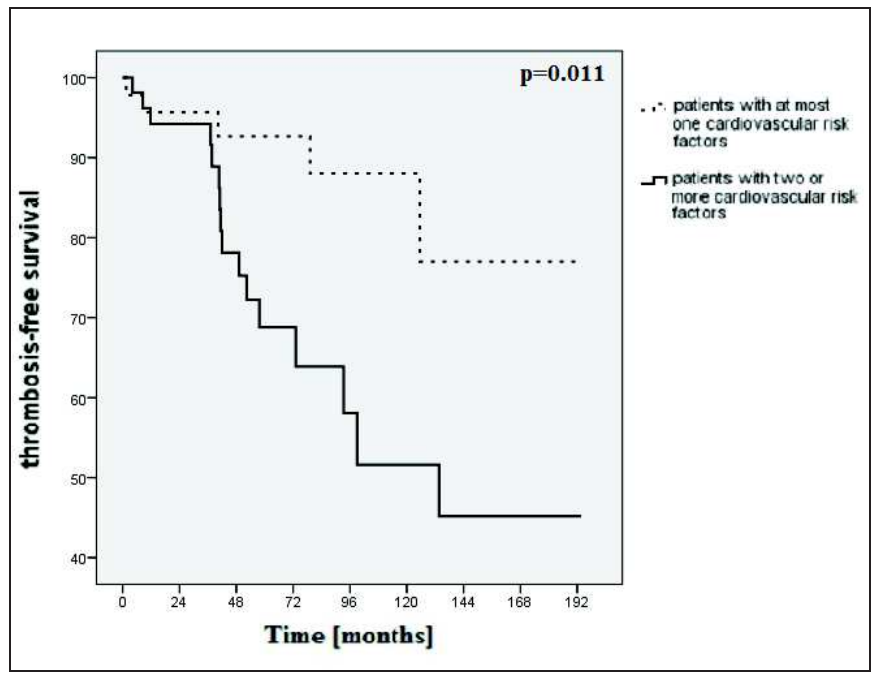

Figure 2. Probability of thrombosis-free survival during haematological follow-up in subgroups of polycythaemia vera patients with at most one cardiovascular risk factor and with two or more cardiovascular risk factors

In another complex overview, similar results were obtained for $\mathrm{CV}$ risk factors in female patients with MPDs; however, the study population for these analyses was defined as the sum of female patients with ET or PV [25]. 


\subsection{SYSTEMIC MASTOCYTOSIS}

\subsubsection{GENERAL CONSIDERATIONS AND BACKGROUND OF THE SYSTEMIC} MASTOCYTOSIS STUDY

Mastocytosis represents one of the eight subcategories of MPNs in the 2008 WHO classification of myeloid neoplasms and acute leukaemia. It is an orphan disease characterized by the pathological accumulation of morphologically and immunophenotypically abnormal mast cells in one, two, or more organ systems. Organ systems most often involved are the bone marrow, skin, liver, and gastrointestinal tract [26]. The clinical presentation of mastocytosis is heterogeneous, ranging from skin-limited disease (cutaneous mastocytosis $[\mathrm{CM}]$ ) affecting particularly children that may spontaneously regress to varying degrees of extracutaneous involvement (SM) generally seen in adults that may be associated with multiorgan dysfunction and a reduced survival [27-30].

The clinical course of SM varies from an asymptomatic form (indolent SM [ISM]) to a highly progressive type (aggressive SM [ASM]) or even mast cell leukaemia (MCL) [31].

The advanced features of the 2008 WHO classification are reflected not only in its novel, molecular-based nomenclature and clear diagnostic criteria supporting the differentiation between each subcategory but also in its high prognostic relevance for SM. In the ever largest clinical trial to validate this correlation, Lim et al. found that, compared to subjects with ASM or SM with an associated clonal haematological non-mast cell lineage disease (SM-AHNMD), ISM patients had a significantly better prognosis in terms of overall survival and leukaemia-free survival. Furthermore, there was no significant difference between the life expectancy of ISM patients and the age- and sex-matched American (USA) population for the appropriate time period, based on the date of diagnosis [27].

According to their clinicopathological features, the revised 2008 WHO classification distinguishes several subcategories within the group of MCDs.

As per the 2008 WHO criteria outlined in Tables 12-13, the following categories of SM are defined: ISM, SM-AHNMD, ASM, and MCL. The diagnosis of SM can only be confirmed after the identification of morphological, immunophenotypic, and/or mutational characteristics of the neoplastic mast cells in an extracutaneous tissue, usually in the bone marrow. In addition, the WHO classification includes $\mathrm{CM}$ and rare, localized mast cell tumours, namely mast cell sarcoma (MCS) and extracutaneous mastocytoma. These entities do not fall under the category of SM and were therefore not included in our research aimed exclusively at the investigation of SM cases. 
Table 12. WHO classification of mastocytosis [13, 30, 32]

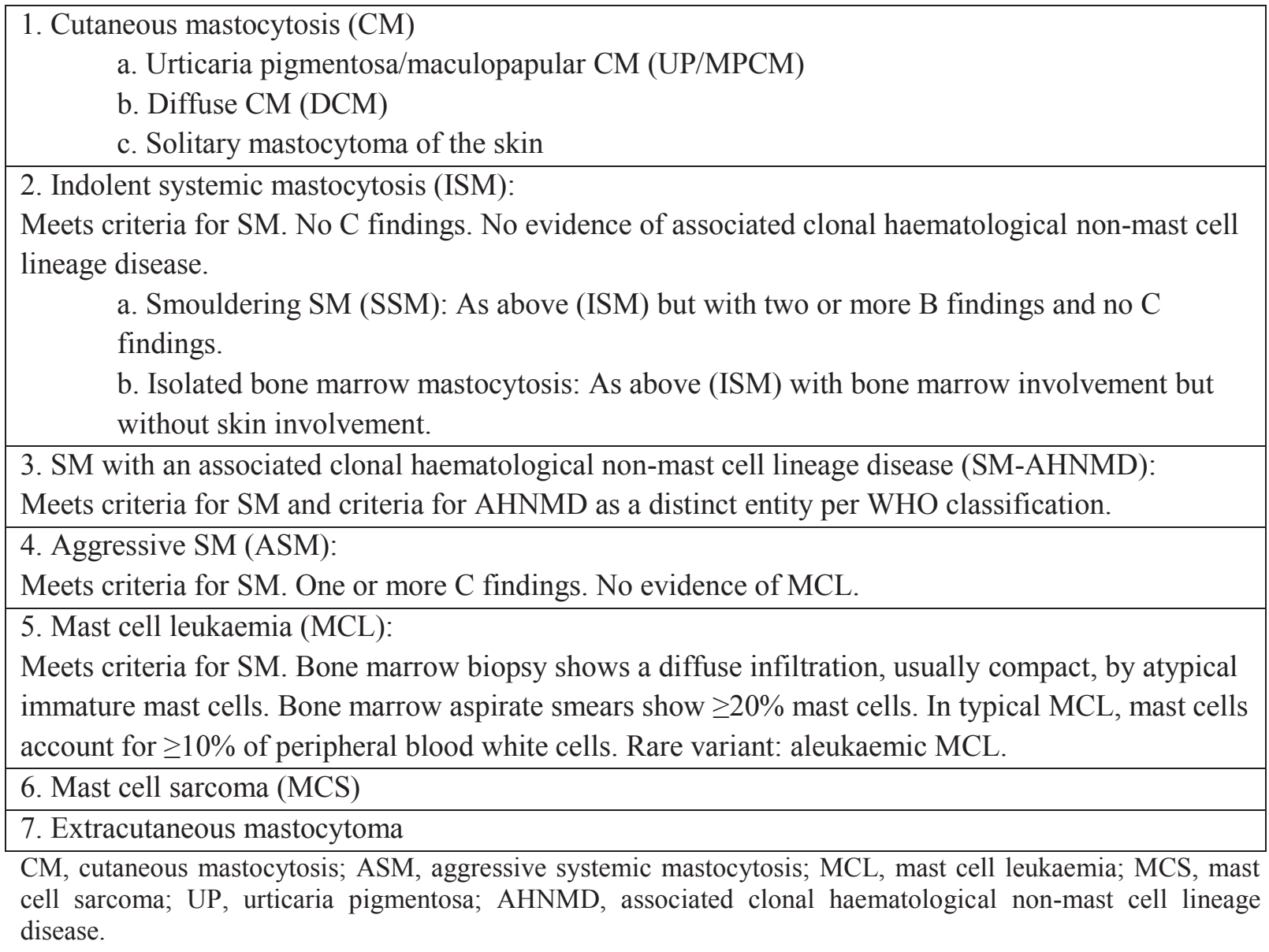

Table 13. $B$ and $C$ findings in advanced mastocytosis

B findings

1. Bone marrow biopsy showing $>30 \%$ infiltration by mast cells (focal, dense aggregates) and/or serum total tryptase level $>200 \mathrm{ng} / \mathrm{mL}$.

2. Signs of dysplasia or myeloproliferation in non-mast cell lineage(s), but insufficient criteria for definitive diagnosis of a haematopoietic neoplasm (AHNMD), with normal or slightly abnormal blood counts.

3. Organomegaly: palpable hepatomegaly, splenomegaly, or lymphadenopathy (on CT or US $>2 \mathrm{~cm}$ ) without impaired organ function.

C findings

1. Cytopenia(s) $\left(\mathrm{ANC}<1.0 \times 10^{9} / \mathrm{L}, \mathrm{Hb}<10 \mathrm{~g} / \mathrm{dL}\right.$, or PLT $\left.<100 \times 10^{9} / \mathrm{L}\right)$ but no obvious non-mast cell haematopoietic malignancy.

2. Palpable hepatomegaly with impairment of liver function, ascites, and/or portal hypertension.

3. Skeletal involvement with large osteolytic lesions and/or pathological fractures.

4. Palpable splenomegaly with hypersplenism.

5. Malabsorption with weight loss due to gastrointestinal mast cell infiltrates.

AHNMD, associated clonal haematological non-mast cell lineage disease; ANC, absolute neutrophil count; CT: computed tomography; Hb, haemoglobin; PLT, platelet; US, ultrasonography. 
The diagnosis of SM can be made when the major criterion and $\geq 1$ minor criterion, OR $\geq 3$ minor criteria are present (Table 14).

Table 14. Schematic overview of the 2008 WHO diagnostic criteria of systemic mastocytosis [30] [33]

\begin{tabular}{|l|}
\hline Major criterion \\
\hline $\begin{array}{l}\text { Multifocal, dense infiltrates of mast cells ( } \geq 15 \text { mast cells in aggregates) detected in bone } \\
\text { marrow and/or other extracutaneous organs }\end{array}$ \\
\hline Minor criteria \\
\hline $\begin{array}{l}\text { a. }>25 \% \text { of the mast cells in bone marrow or other extracutaneous organ(s) show an abnormal } \\
\text { morphology in bone marrow smears or in histologies }\end{array}$ \\
\hline b. KIT mutation at codon 816 in extracutaneous organ(s) \\
\hline c. Mast cells in bone marrow express CD2 and/or CD25 \\
\hline d. Serum total tryptase $>20 \mathrm{ng} / \mathrm{ml}$ (does not count in patients with AHNMD-type disease) \\
\hline
\end{tabular}

AHNMD, associated clonal haematological non-mast cell lineage disease.

Although there is no internationally accepted, universal definition for orphan diseases, the different criteria are common in their use of prevalence rates. The prevalence threshold varies widely between the European Union, the USA, and Japan. According to the definition of the European Committee for Orphan Medicinal Products, severe or life-threatening conditions with a prevalence of less than 5:10,000 are considered as orphan diseases [34, 35]. Up to now, there are only very limited data on the incidence and prevalence of mastocytosis. Therefore, ECNM has established a registry to collect information from a number of patients suffering from this rare disease [36]. Within this program, an incidence of 5 to 10 cases per $1,000,000$ person-years was obtained [33].

The prevalence of mastocytosis in Central Europe is estimated at 0.5-1.0:10,000 [37]. Population-based epidemiological data and local/regional data on ISM have been reported from Denmark and The Netherlands (Groningen) but ECNM is still collecting data from ten European countries [36, 38, 39]. ISM is the most frequent subtype of SM in adults. It is predominated by cutaneous manifestations (UP) but recurrent systemic symptoms (e.g. flushing, palpitations, muscle cramps, abdominal pain, diarrhoea, bone pain) related to mast cell degranulation and mediator release and/or allergies or anaphylaxis may also occur. Factors which may lead to mast cell activation include heat, cold, stress (physical or emotional), medications, insect bites, and food or are idiopathic. The symptoms may have a strong negative impact on the quality of life while anaphylactic reactions can be severe or even fatal $[26,38]$. 
On the contrary, symptoms in ASM (e.g. cytopenia, ascites, malabsorption, or osteolytic skeletal lesions) arise from organ dysfunction due to mast cell infiltration. SM-AHNMD is characterized by the presence of another clonal haematological disease such as myelodysplastic syndrome (MDS), myeloid leukaemia or another MPN, or non-Hodgkin lymphoma concomitant to SM [26, 38]. Mast cells are tissue resident cells of HSC origin. The differentiation and survival of mast cells is mainly regulated by the activation of KIT by its ligand stem cell factor $[40,41]$ Most of the adult patients suffering from mastocytosis, regardless of disease subtype, harbour the somatic activating mutation of the oncogenic receptor tyrosine kinase KIT gene (exon 17, D816V) [33, 42-45] The KIT D816V mutation, which is found in up to $85 \%$ of all SM patients, is of great pathogenetic and diagnostic relevance [26, 43-45]

\subsubsection{PATIENTS AND DATA COLLECTION}

Between 2001 and 2013, a total of 35 patients were diagnosed with SM (20 males, 15 females; median age: 57 years [range: $31-85$ years]) in our centre: 14 with ISM, 15 with SM-AHNMD, and 6 with ASM subtypes, respectively (Table 15). In the investigated period, no other MCD entities like MCL, extracutaneous mastocytoma, or MCS occurred. Out of all registered cases, only those who strictly fulfilled the 2008 WHO criteria for SM (major criterion and $\geq 1$ minor criterion, $\mathrm{OR} \geq 3$ minor criteria) were considered to be enrolled in our research.

In Hungary, serum tryptase determination is currently not available; however, all our presented cases met the 2008 WHO criteria, even without known tryptase levels. The haematological management of each particular patient used to be based on the current treatment standards available at time of diagnosis and therapy initiation [32, 46-53]. 
Table 15. Summary of the main demographic and disease-related characteristics of the investigated sytemic mastocytosis study population by disease subtype

\begin{tabular}{|c|c|c|c|}
\hline Characteristics & ISM & SM-AHNMD & ASM \\
\hline \multicolumn{4}{|l|}{ Main demographic characteristics } \\
\hline Patients (N) & 14 & 15 & 6 \\
\hline Males (N) & 8 & 7 & 4 \\
\hline Females $(\mathrm{N})$ & 6 & 8 & 2 \\
\hline Median age at diagnosis (years) (range) & $55(31-81)$ & $57(34-72)$ & $65(54-85)$ \\
\hline Median follow-up (months) (range) & $50.5(5-240)$ & $25(1-104)$ & $20.5(2-35)$ \\
\hline \multicolumn{4}{|l|}{ Disease-related characteristics } \\
\hline Associated haematological disease (N) & - & $\begin{array}{l}\text { MDS (3) } \\
\text { AML (3) } \\
\text { MF (2) } \\
\text { ET (1) } \\
\text { PV (1) } \\
\text { CML (1) } \\
\text { iHES (1) } \\
\text { MM (1) } \\
\text { PTCL (1) } \\
\text { B-ALL (1) }\end{array}$ & - \\
\hline Urticaria pigmentosa $(\mathbf{N})$ & $8 / 14$ & - & - \\
\hline $\begin{array}{l}\text { Mediator-related symptoms (N) } \\
\text { skin (flush, pruritus) } \\
\text { gastrointestinal (diarrhoea) } \\
\text { cardiovascular (palpitation, dizziness, syncope) } \\
\text { neurological } \\
\text { anaphylaxis }\end{array}$ & $\begin{array}{l}5 / 14 \\
3 / 14 \\
2 / 14 \\
1 / 14 \\
2 / 14\end{array}$ & $\begin{array}{l}- \\
- \\
- \\
- \\
-\end{array}$ & $\begin{array}{l}2 / 6 \\
- \\
- \\
1 / 6 \\
-\end{array}$ \\
\hline $\begin{array}{l}\text { Coexistent allergy (N) } \\
\text { (inhalation, nutritive, drug, insect venom) }\end{array}$ & $3 / 14$ & - & - \\
\hline $\begin{array}{l}\text { Constitutional symptoms (N) } \\
\text { (generalized weakness, fatigue, sweats, chills, } \\
\text { arthralgia, myalgia) }\end{array}$ & $1 / 14$ & $1 / 15$ & $6 / 6$ \\
\hline $\begin{array}{l}\text { Organ damage/Organopathy (N) } \\
\text { Hepatomegaly / } \\
\text { *hepatomegaly with elevated alkaline phosphatase } \\
\text { Splenomegaly } \\
\text { Adenopathy } \\
\text { Osteopenia/osteoporosis/osteolysis }\end{array}$ & $\begin{array}{l}2 / 14 \\
1 / 14^{*} \\
- \\
1 / 14 \\
3 / 14\end{array}$ & $\begin{array}{l}3 / 15 \\
2 / 15 \\
1 / 15 \\
- \\
\end{array}$ & $\begin{array}{l}6 / 6^{*} \\
3 / 6 \\
3 / 6 \\
3 / 6\end{array}$ \\
\hline
\end{tabular}

AML, acute myeloid leukaemia; ASM, aggressive systemic mastocytosis; B-ALL, B-cell acute lymphoblastic leukaemia; CML, chronic myeloid leukaemia; ET, essential thrombocythaemia; iHES, idiopathic hypereosinophilic syndrome; ISM, indolent systemic mastocytosis; MDS, myelodysplastic syndrome; MF, myelofibrosis; MM, multiple myeloma; PTCL, peripheral T-cell lymphoma; PV, polycythaemia vera; SM-AHNMD, systemic mastocytosis with an associated clonal haematological non-mast cell lineage disease. 


\subsubsection{STATISTICAL ANALYSES}

Evaluation of the survival probability in systemic mastocytosis

The survival probability in the various SM subgroups (ISM, AHNMD, ASM) was estimated by Kaplan-Meier analyses. Calculated patient survival rates were compared to the expected survival data of age- and sex-matched Hungarian population controls obtained from the Hungarian Central Statistical Office. Age- and sex-matched survival statistics were retrieved from life tables based on a population of 1,000 newborns [22]. For each year, the incidence rate was calculated as the number of new cases divided by the mid-year population size. The latter was obtained as the mean of the population sizes on $1^{\text {st }}$ January of the relevant year and the next year. The cumulative incidence for 13 years was computed as $C I=1-e^{-\sum_{i=1}^{13} I R_{i} \cdot t_{i}}$

, where $I R_{i}$ denotes the yearly incidence rates from the first to the thirteenth year, and $t_{i}$ denotes the length of each time period which is one year in this case for all the 13 periods.

\subsubsection{LABORATORY METHODS}

Our haematological centre - operating as an integrated part of the $2^{\text {nd }}$ Department of Internal Medicine and Cardiology Centre - is a regional haematological diagnostic and treatment centre catering for a population of approx. 1,103,463 inhabitants in south-eastern Hungary (Figure 3). Our data were in part retrieved from the outpatient and inpatient database of our centre sorted by ICD code and in part obtained from bone marrow biopsy reports released by the Laboratory of Tumour Pathology and Molecular Diagnostics, Szeged. All paediatric patients with $\mathrm{CM}$ and adult patients presenting with skin lesions who refused bone marrow biopsy were not included in our study.

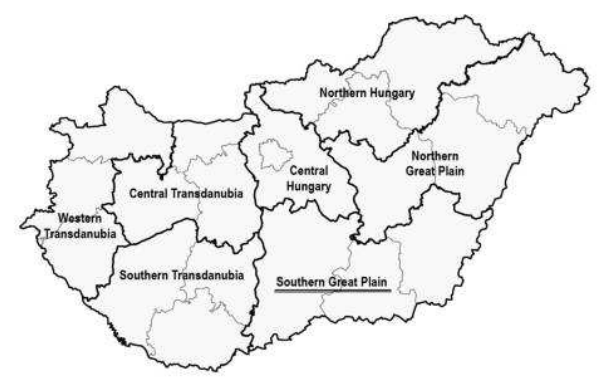

Figure 3. Geographical location of our regional centre catering for the population of three south-eastern counties of Hungary (South Great Plain region).

Source: http://www.ksh.hu/regional atlas counties?lang=en 
All bone marrow test results released since 2001 were screened for SM and re-assessed by morphological, immunohistochemical and molecular (PCR) methods, according to the $2008 \mathrm{WHO}$ criteria.

We considered only those cases as SM which strictly fulfilled the relevant 2008 WHO criteria (major criterion and $\geq 1$ minor criterion, OR $\geq 3$ minor criteria). In Hungary, serum tryptase determination is currently not available; however, all our presented cases met the 2008 WHO criteria, even without known tryptase levels. C-KIT mutation analysis is routinely performed on every bone marrow biopsy sample evaluated at our centre. C-KIT mutations detected by PCR method in this patient population were confirmed by Sanger sequencing. Out of all detected cases, only those were incuded in our study database which strictly complied with the relevant 2008 WHO criteria (major criterion and $\geq 1$ minor criterion, OR $\geq 3$ minor criteria).

\section{Immunohistochemistry}

Bone marrow trephine biopsies were fixed in neutral buffered formaldehyde supplemented with methanol and glucose (Schaffer's fixative), decalcified in $12.5 \%(\mathrm{w} / \mathrm{v})$ pH 7.0 EDTA solution (Sigma-Aldrich), and embedded into paraffin. The immunohistochemical reactions were executed on 2-4 $\mu \mathrm{m}$ thick formaldehyde-fixed and paraffin-embedded (FFPE) sections waxed in xylene and graded ethanol, and pretreated by heat-induced antigen retrieval. The following primary antibodies were used: anti-CD117 (Dako, Denmark A/S), anti-CD25, anti-mast cell tryptase, anti-CD68 (Leica Biosystems/Novocastra), and anti-phospho-STAT5 (Santa Cruz Biotechnology, USA). Detection was carried out with Novolink polymer kit (Leica Biosystems/Novocastra) according to the manufacturer's instructions while nuclear staining was completed with Mayer's haematoxylin.

\section{DNA isolation, PCR amplification, and DNA sequencing}

Molecular tests were performed on crude DNA lysates made from FFPE tissue sections. Briefly, ten pieces of paraffin sections of $10 \mu \mathrm{m}$ thickness per each bone marrow trephine biopsy sample were placed into sterile $1.5 \mathrm{ml}$ Eppendorf tubes, mounted with $100 \mu 1$ lysis buffer (50 mM Tris- $\mathrm{HCl}, 1.5 \mathrm{mM} \mathrm{MgCl}_{2}, \mathrm{pH} 8.0$ ) containing $10 \mu 1$ proteinase $\mathrm{K}$ (PK) solution (20 mg/ml, MBI Fermentas Life Sciences), centrifuged at 13,000 rpm for $3 \mathrm{~min}$, and incubated at $56{ }^{\circ} \mathrm{C}$ for $18 \mathrm{~h}$. Afterwards, PK was inactivated at $96{ }^{\circ} \mathrm{C}$ for $15 \mathrm{~min}$ and centrifuged at 13,000 rpm for $3 \mathrm{~min}$. The retrieved supernatant was used as template in 1:10- 
1:20 dilution. PCR amplification was carried out in $25 \mu \mathrm{l}$ reaction mixture in an Eppendorf Mastercycler ${ }^{\circledR}$ gradient thermal cycler. PCR parameters were as follows: $100 \mu \mathrm{M}$ dNTP (MBI Fermentas Life Sciences), $1.75 \mathrm{mM} \mathrm{MgCl} 2,25 \mathrm{pmol} / \mu 1$ of each primer, $2 \mu 1$ DNA template, and 1.5 IU recombinant Taq polymerase (MBI Fermentas Life Sciences) per reaction. The following primers were used: C-KIT-outer-Fo 5'-GCCAGAAATATCCTCCTTACTCA-3', C-KIT-allele-specific-Fo 5'-GTGATTTTGGTCTAGCCAGCKT-3', C-KIT-Re 5'-GTTGAAACTAAAAATCCTTTGCAGGAC-3'. The temperature and timing parameters of the cycles were as follows: denaturation at $95{ }^{\circ} \mathrm{C}$ for $30 \mathrm{~s}$, annealing at $56{ }^{\circ} \mathrm{C}$ for $30 \mathrm{~s}$, extension at $72{ }^{\circ} \mathrm{C}$ for $30 \mathrm{~s}$, last extension at $72{ }^{\circ} \mathrm{C}$ for $10 \mathrm{~min} . \mathrm{PCR}$ products were run on $12.5 \%$ polyacrylamide gel using the Mini PROTEAN ${ }^{\circledR}$ Tetra Cell (Bio-Rad Laboratories) electrophoresis set and visualized with $\mathrm{AgNO}_{3}$ staining. This primer set generated a 153-basepair-long outer PCR product used as reaction control and a 111-basepair-long mutation-specific product. PCR products of expected size were Sanger sequenced using Applied Biosystems ${ }^{\circledR} 3500 \mathrm{DX}$ series genetic analyser and evaluated with the free Sequence Scanner software (v1.0). The sequences obtained were run against the BLAST database.

In one single case [54], we screened our patient by Sanger sequencing for the most frequently reported KIT mutations in exons 9,11 , and 17 in a close co-operation with the $1^{\text {st }}$ Department of Pathology and Experimental Cancer Research, Semmelweis University, Budapest [54].

\subsubsection{RESULTS}

CLINICOPATHOLOGICAL FINDINGS ALONG WITH BONE MARROW HISTOLOGICAL FEATURES, MOLECULAR CHARACTERISTICS, AND LABORATORY PARAMETERS AT PRESENTATION IN A LARGE COHORT OF SYSTEMIC MASTOCYTOSIS PATIENTS (Aim II.a)

In the ISM group, bone marrow biopsy analysis revealed ISM in 14 patients ( 8 males and 6 females) with a median age of 55 years (range: $31-81$ years). The median duration of follow-up was 50.5 months (range: 5-240 months). Cutaneous manifestation (UP) was detected in $57 \%(8 / 14)$ of the patients while mediator-related symptoms occurred in $28 \%$ $(4 / 14)$ of them. $78 \%(11 / 14)$ of the ISM patients were positive for KIT D816V mutation.

A total of 15 patients ( 7 males and 8 females; median age: 57 years [range: $34-$ 72 years]) were diagnosed with AHNMD. The median follow-up time in this subgroup was 25 months (range: 1-104 months). Bone marrow biopsy was done as required by signs of the associated neoplasm such as bone lesions or clinically significant peripheral blood count abnormalities, e.g. eosinophilia or elevated or decreased WBC or PLT counts. This subtype of 
SM was associated with MDS or acute myeloid leukaemiain three cases each, with MF in two cases, or with ET, PV, CML, HES, multiple myeloma, peripheral T-cell lymphoma, or B-cell acute lymphoblastic leukaemia in one case each. In these patients, no cutaneous lesions or mediator-related symptoms were observed. KIT D816V mutation positivity was detected in $80 \%(12 / 15)$ of the patients.

ASM was diagnosed in 6 patients (4 males and 2 females) with a median age of 65 years (range: 54-85 years). The median follow-up was 20.5 months (range: 2-35 months). Bone marrow biopsy was performed for hepatosplenomegaly with or without pancytopenia/anaemia/eosinophilia and weight loss. All ASM patients presented with at least one C finding, as defined by the 2008 WHO criteria (marked cytopenia, osteolysis with or without pathological fractures, ascites and elevated liver enzymes, malabsorption with hypoalbuminaemia, palpable splenomegaly with hypersplenism). Cutaneous lesions were detected in $33 \%(2 / 6)$ of the patients. Mediator-related symptoms occurred in one case. KIT D $816 \mathrm{~V}$ mutation positivity was confirmed in $83 \%(5 / 6)$ of the patients.

Clinical symptoms, immunophenotypes as well as PCR and Sanger sequencing results for each patient are summarized in Supplement 2 to this dissertation (Appendix III) and the subgroup distribution (ISM; AHNMD, ASM) and detailed characteristics of these patients have been recently published in Supplement 1 (Appendix III) to the Clinical and Molecular Diagnostic Evaluation of Systemic Mastocytosis in the South-Eastern Hungarian Population Between 2001-2013 - A Single-centre Experience by Imelda Marton et al. [55].

\section{FREQUENCY OF KIT D816V MUTATION (Aim II.b)}

KIT D $816 \mathrm{~V}$ mutation positivity was detected in $78 \%$ (11/14) of ISM patients, $80 \%$ $(12 / 15)$ of AHNMD patients and 83\% (5/6) of ASM patients, respectively. Main patient characteristics including bone marrow biopsy test PCR and Sanger sequencing results results are presented in Supplement 2 to this dissertation (Appendix III ) [55].

\section{LIFE EXPECTANCY IN SYSTEMIC MASTOCYTOSIS (Aim II.c)}

Overall disease-specific survival of SM patients was analyzed by Kaplan-Meier method and is demonstrated in Figure 4. 


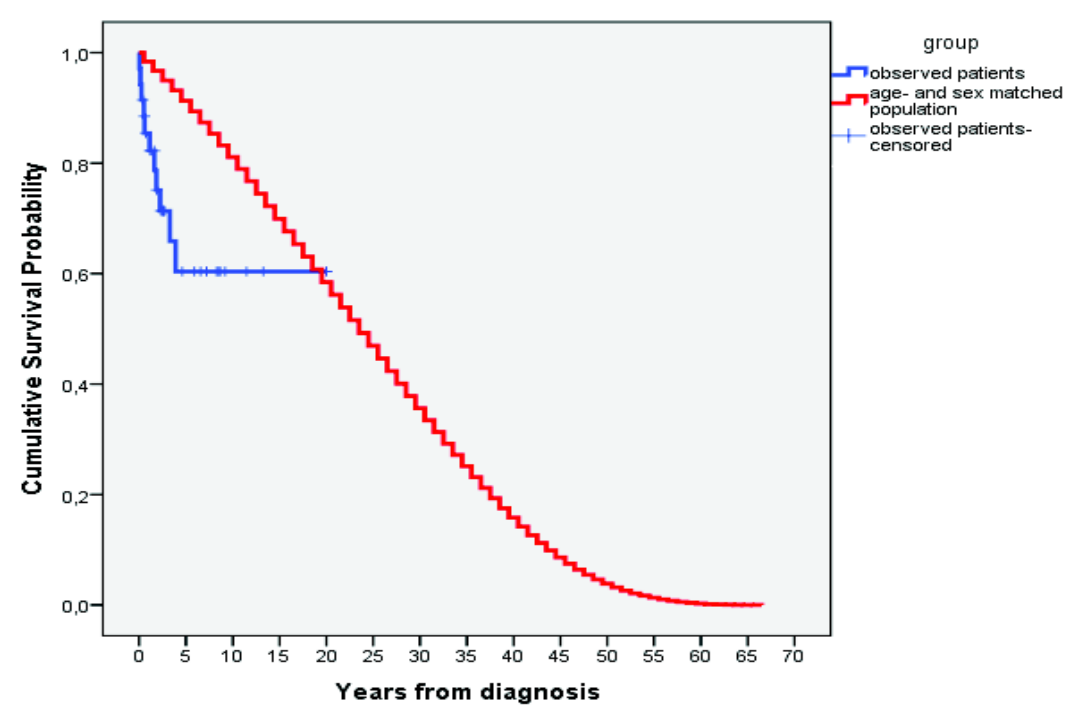

Figure 4. Kaplan-Meier survival curve demonstrating cumulative survival probability of patients with systemic mastocytosis. The survival observed in SM patients (blue) is compared to the expected survival of the age- and sex-matched Hungarian population (red).

Similarly, survival data for each SM subtype were also generated by Kaplan-Meier analysis and are presented in Figure 5.

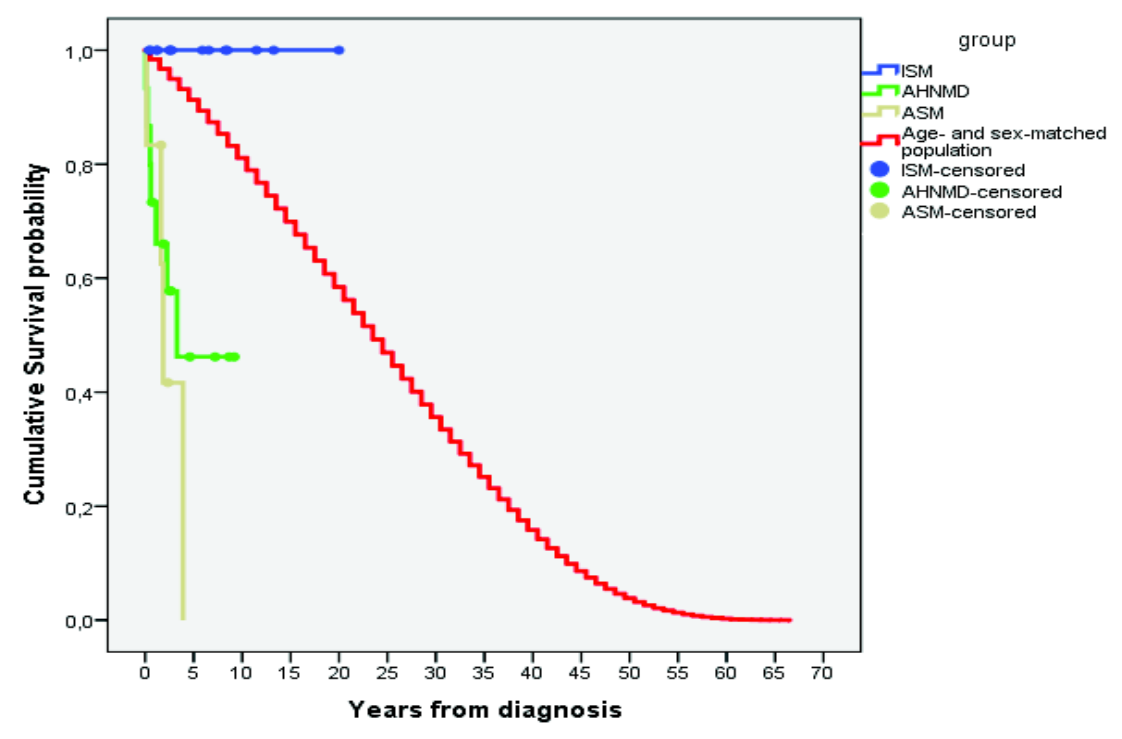

Figure 5. Survival of systemic mastocytosis patients by disease subtype. Kaplan-Meier survival rates of SM patients classified by disease subtype - ISM (blue), AHNMD (green), and ASM (yellow) - were compared to the expected survival of the age- and sex-matched Hungarian population (red). 
The median survival in the ASM group was 1.73 years while the survival time of the AHNMD patients did not reach a median during follow-up. None of the patients died during the follow-up period in the ISM subpopulation. The median survival for the age- and sexmatched control population was 23.5 years. The comparison of the survival curves using Mantel-Cox, Breslow and Tarone-Ware tests uniformly resulted in a p-value of 0.000 indicating significantly different survival patterns in the evaluated SM subgroups.

Moreover, it is important to emphasize that our research identified an uncommon case in the investigated patient population. In our case report [54], we demonstrated a female ISM patient with recurrent cutaneous symptoms and a follow-up time of 27 years. This case illustrates that in selected cases, imatinib mesylate could be a good choice to achieve a reduction of skin lesions, like in this KIT D816V-negative patient. The case is presented in details in Appendix $I V$ to this dissertation as the publication of Imelda Marton et al.: Therapeutic challenge during the long-term follow-up of a patient with indolent systemic mastocytosis with extensive cutaneous involvement.

\section{CUMULATIVE INCIDENCE OF SYSTEMIC MASTOCYTOSIS (Aim II.d)}

Our regional diagnostic and treatment centre receives SM patients from the population of south-eastern Hungary, representing a total of 1,103,463 inhabitants [56]. These data allowed us to calculate the cumulative incidence of SM for 13 years in the general population, which proved to be $0.27 / 10,000$ in this region.

Four patients (Cases 2, 13, 29, and 35) were excluded from this estimation of the cumulative incidence: although they were diagnosed in our centre, were inhabitants of another administrative region of Hungary.

\subsection{HYPEREOSINOPHILIC SYNDROME}

\subsubsection{GENERAL CONSIDERATIONS AND BACKGROUND OF THE} HYPEREOSINOPHILIC SYNDROME STUDY

Traditionally, peripheral blood eosinophilia was classified as mild (absolute eosinophil

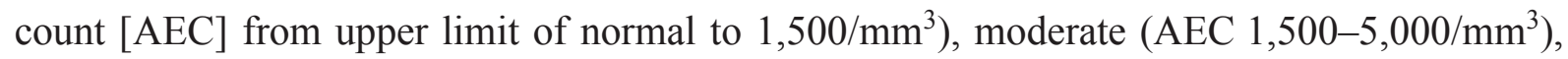
and severe $\left(\mathrm{AEC}>5,000 / \mathrm{mm}^{3}\right)$. Hypereosinophilia defined as AEC $>1,500 / \mathrm{mm}^{3}$ may be asssociated with tissue damage [57-60]. The current definitions and criteria of eosinophilic disorders and related syndromes are often overlapping, both with each other and within the area of several disciplines like pathology, haematology, immunology, and allergology. Hence, 
the establishment of multidisciplinary definitions along with refined criteria for the various forms of hypereosinophilia has become essential [61]. If any secondary cause of eosinophilia can be excluded, the condition is classified as either clonal or idiopathic primary eosinophilia, depending on the presence or absence of a molecular, cytogenetic, or histological evidence for a myeloid malignancy [59].

The classification of eosinophilic diseases has been revised by the updated 2008 WHO scheme (Table 1). CEL-NOS is one of the eight subcategories within MPNs. Reflecting the growing number of recurrent, molecularly defined primary eosinophilias, a new major category of "myeloid and lymphoid neoplasms with eosinophilia and abnormalities of PDGFRA, PDGFRB, or FGFRI" has been generated [62]. Idiopathic HES represents a distinct entity (Table 16) .

The rearranged $P D G F R A / B$ and $F G F R 1$ fusion genes encode constitutively activated tyrosine kinases. Out of them, PDGFRA/B-rearranged neoplasms with eosinophilia are imatinibsensitive. Therefore, this classification has a direct therapeutical relevance, indicating imatinib as the appropriate definitive treatment in these conditions. The most common cytogenetic alteration in myeloid and lymphoid neoplasms accompanied by eosinophilia and abnormalities of PDGFRA is FIP1L1-PDGFRA gene fusion first described by Cools et al. in 2003 [63].

Table 16. Schematic overview of 2008 WHO classification of eosinophilic disorders [64]

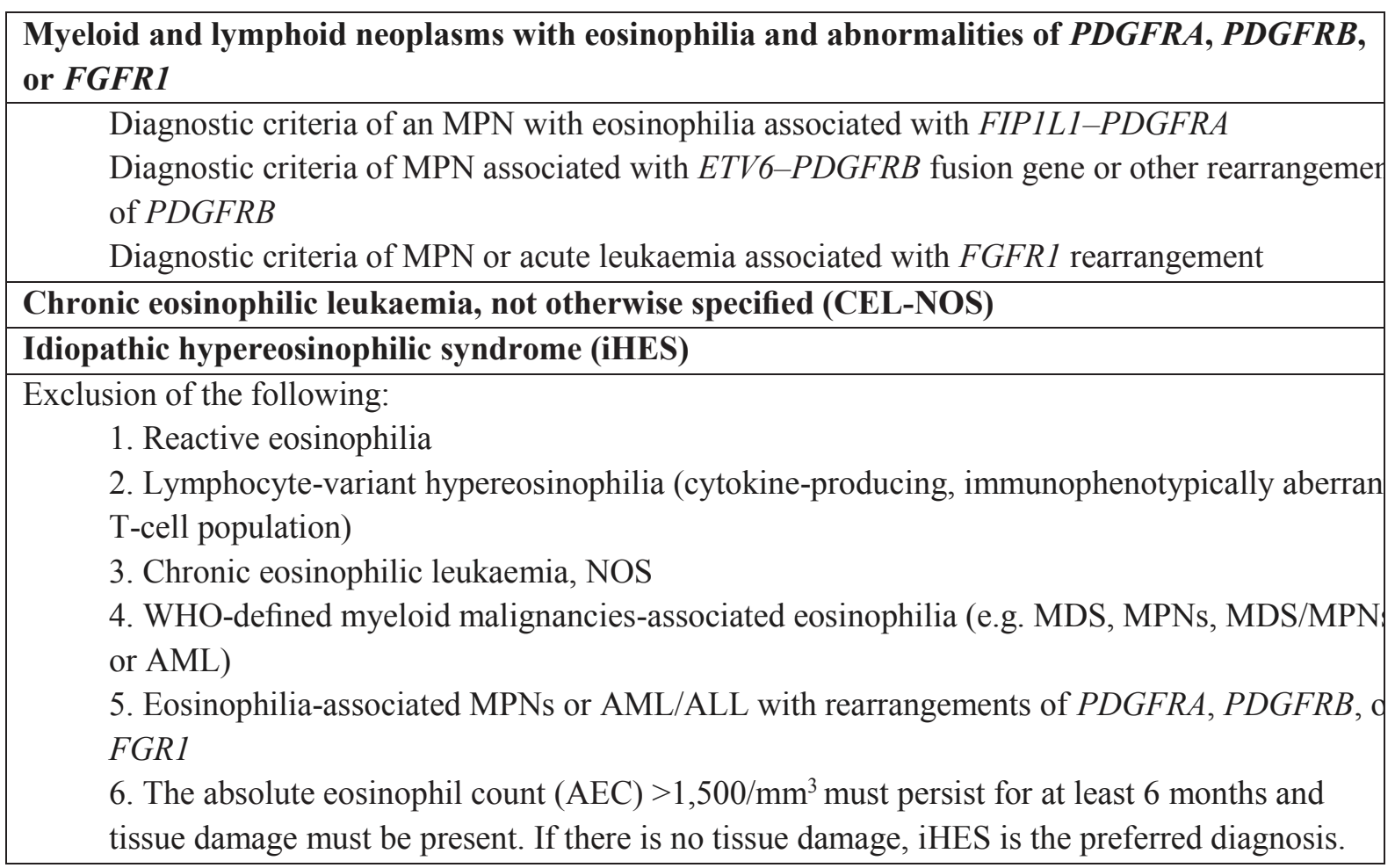


Laboratory evaluation of primary hypereosinophilia should start with screening of peripheral blood or bone marrow for FIPIL1-PDGFRA gene fusion either by real-time PCR (RT-PCR) or interphase/metaphase fluorescence in situ hybridization (FISH). Abnormalities of PDGFRB or FGFR 1 could be identified by conventional cytogenetic analysis or FISH [62]. Studies conducted in developed countries indicate that FIP1L1-PDGFRA fusion occurs in approximately $10 \%$ of patients with hypereosinophilia of unknown aetiology [65-67]. While imatinib mesylate (imatinib) administered in lower doses than in CML and as first-line treatment for patients with abnormalities of $P D G F R A / B$ is highly effective to reach and maintain molecular remission, patients with FGFR1 fusions are resistant to this drug and have a poor prognosis [62].

The WHO definition of HES is based on the historical criteria outlined by Chusid et al. in 1975. According to these, the diagnosis of HES can be established if AEC $>1,500 / \mathrm{mm}^{3}$ persists for $>6$ months and tissue damage is present without secondary causes of eosinophilia (such as parasitic infection, drugs, connective tissue disorders, vasculitis, malignancies, or allergies) [68]. However, the criterion of a 6-month-long duration of elevated AEC may be abandoned as the primary goal is the rapid correction of hypereosinophilia and the prevention of organ failure by an early and effective treatment. In the absence of organ damage, the preferred term is "idiopathic hypereosinophilia" instead of HES [69]. The proper classification is fairly difficult as, on the one hand, there is a considerable overlapping between the various conditions with hypereosinophilia and, on the other hand, the nomenclature itself has been continuously debated for decades.

The most common signs and symptoms in HES are asthenia and fatigue (26\%), cough $(24 \%)$, dyspnoea (16\%), myalgia or angiooedema (14\%), rash or fever $(12 \%)$, and rhinitis (10\%) [61]. Typical laboratory findings include leukocytosis $\left(\geq 20,000-30,000 / \mathrm{mm}^{3}\right)$ with peripheral eosinophilia up to a level of $30-70 \%$ [70]. The clinical manifestation of HES is diverse ranging from an asymptomatic form to a progressive course with severe symptoms and multi-organ involvement. Sustained eosinophilia may affect all tissues and organ systems. Most frequently reported complications include dermatological, neurological, pulmonary, cardiac, and - less commonly - gastrointestinal conditions [70-73].

The major cause of morbidity and mortality is eosinophilic myocarditis developing secondary to endomyocardial fibrosis and restrictive cardiomyopathy. Loeffler's endocarditis with eosinophilic infiltration represents the prototype of cardiac manifestations in HES [7]. The clinical course of Loeffler's endocarditis typically progresses in three - necrotic, thrombotic, 
and fibrotic - stages into endomyocardial fibrosis and restrictive endomyocardyopathy as endstage [74]. Many hypotheses have been proposed to explain the pathomechanism of cardiac and other organ dysfunctions in HES. The primary target of the tissue damage initiating local thrombosis is endothelial cells of the endocardium [48]. Major basic protein released from eosinophilic granules may exert endothelial cell damage while eosinophilic cationic protein may be responsible for the hypercoagulable state. The direct toxic and procoagulant effect of eosinophilic derivatives may contribute to the development of thrombosis and cardiac embolism [58-61, 71]This may explain central nervous system (CNS) injury: not only eosinophil-induced endothelial dysfunction but also cardiac microembolization might play an important role in the development of cerebral infarction and neurological dysfunction. Toxic effects of released eosinophilic basic proteins may initiate endomyocardial necrosis which occurs in the early stages and usually remains subclinical. After 4-6 weeks of disease onset the excessive release of tissue factor from damaged tissue cells and eosinophils may lead to endomyocardial fibrosis [59, 75-77]. Case series published after the first description of the disease reported very poor prognosis and short survival in HES, primarily due to the advanced state of the disease and the congestive heart failure. In a report by Chusid et al., median survival was as short as 9 months while 3 -year survival was only $12 \%$ [68]. A later publication on 40 HES patients talked about a 5-year survival rate of $80 \%$, decreasing to $42 \%$ at 15 years [73].

\subsubsection{PATIENTS AND DATA COLLECTION IN THE HYPEREOSINOPHILIC SYNDROME POPULATION}

The diagnosis of idiopathic HES can only be established after the exclusion of all primary and secondary causes of hypereosinophilia and lymphocyte-variant hypereosinophilia [69]. We evaluated 10 iHES patients with hypereosinophilia fulfilling the 2008 WHO criteria but without any secondary causes or underlying clonal disease. All patients were asymptomatic; none of them had a known Loeffler's endocarditis. Cytogenetic, FISH, and molecular analyses of FIP1L1-PDGFRA were negative for all subjects in this population of 10 iHES patients without any underlying disease. Due to the presence of Loeffler's endocarditis and the rare neurological complication, the case of the patient with FIPIL1PDGFRA mutation will be discussed separately. 
Table 17. Summary of the main demographic and disease-related characteristics of the investigated study population with hypereosinophilic syndrome

\begin{tabular}{|c|c|}
\hline Characteristics & HES \\
\hline \multicolumn{2}{|l|}{ Main demographic characteristics } \\
\hline Patients (N) & 10 \\
\hline Males (N) & 7 \\
\hline Females $(\mathrm{N})$ & 3 \\
\hline Median age at diagnosis (years) (range) & $58.1 \pm 13.1$ \\
\hline Median follow-up (months) (range) & $37(2-120)$ \\
\hline \multicolumn{2}{|l|}{ Disease-related characteristics } \\
\hline Red blood cell count $(\mathrm{T} / \mathrm{L})$ & $4.2 \pm 0.5$ \\
\hline Haemoglobin $(\mathrm{g} / \mathrm{L})$ & $126.7 \pm 18.8$ \\
\hline Haematocrit (\%) & $36.9 \pm 5.5$ \\
\hline Platelet count $(\mathrm{G} / \mathrm{L})$ & $276.3 \pm 176.7$ \\
\hline White blood cell count $(\mathrm{G} / \mathrm{L})$ & $16.7 \pm 5.8$ \\
\hline Percentage of blood eosinophils (\%) & $49.0 \pm 16.6$ \\
\hline Absolute eosinophil count (G/L) & $8.7 \pm 4.8$ \\
\hline
\end{tabular}

\subsubsection{STATISTICAL ANALYSES}

All HES patients and their age- and sex-matched healthy controls underwent complete two-dimensional (2D) Doppler echocardiography and 3DSTE. Data of altogether 10 HES patients were compared to matching data of 19 control subjects.

Continuous variables were calculated as mean \pm standard deviation. All statistical tests were two-sided. The cut-off value for statistical significance was set at $p=0.05$. Continuous parameters were compared using unpaired Student's $t$ test while categorical variables were analyzed by chi-square or Fischer's exact test. The correlation was defined by Pearson's correlation coefficient. Statistical evaluations were performed using MedCalc software (MedCalc Inc., Mariakerke, Belgium).

\subsubsection{METHODS - LABORATORY TESTS AND ECHOCARDIOGRAPHY}

Diagnostic bone marrow samples of all patients investigated for hypereosinophilia were evaluated at our local tumour pathology laboratory. Cytogenetic tests were completed at the laboratory for cytogenetics of the $2^{\text {nd }}$ Department of Internal Medicine and Cardiology Centre while FISH assays were performed at the Department of Haematology and Stem Cell Transplantation, United Szent István and Szent László Hospital, Budapest. Molecular genetic tests for FIP1L1-PDGFRA were carried out in a close co-operation with the team of the Laboratory of Molecular Diagnostics, Hungarian National Blood Transfusion Service, Budapest [63]. 


\section{Three-dimensional speckle-tracking echocardiography (3DSTE)}

3DSTE datasets were acquired from apical window using the 1-4 MHz matrix phasedarray transducer (PST-25SX) [78].

Following gain setting optimization, full volume mode was used over six consecutive cardiac cycles during a single breath-hold. Volume data were stored in raw data format for further analysis. LA quantifications were performed using the Artida 3D Wall Motion Tracking software v2.7 (Toshiba Medical Systems, Tokyo, Japan). Each three-dimensional (3D) dataset was displayed in multiple plane views including the apical two- $(\mathrm{AP} 2 \mathrm{CH})$ and four-chamber $(\mathrm{AP} 4 \mathrm{CH})$ views and three short-axis views at different LA levels from the base to the apex. Several reference points on the LA endocardium were set by the examiner in the AP2CH and $\mathrm{AP} 4 \mathrm{CH}$ views. The first points were set at the edge of the septal mitral valve ring where anterior mitral leaflet origins and then markers were placed in a counterclockwise rotation around the LA to the lateral mitral valve ring (to the origin of the posterior leaflet) in the $\mathrm{AP} 4 \mathrm{CH}$ viewing plane. During evaluations, LA appendage and the pulmonary veins were excluded from the LA cavity. Measurements were performed first on AP4CH view and then on $\mathrm{AP} 2 \mathrm{CH}$ view. After detection of the LA myocardial borders at the end-diastolic reference frame, the user could correct the LA shape if it was necessary. The 3D wall motion tracking was then automatically performed through the entire cardiac cycle.

\section{DSTE for left atrial volumetric measurements}

From time curves of global LA volume changes, maximum $\left(\mathrm{V}_{\max }\right)$, minimum LA volumes $\left(\mathrm{V}_{\min }\right)$ and LA volume before atrial contraction $\left(\mathrm{V}_{\text {preA }}\right)$ were measured using the 3D echocardiographic datasets just before mitral valve opening (end-systole), just before mitral valve closure (end-diastole) and at time of P-wave on electrocardiography (early diastole), respectively [78-82] LA function consists of three phases: the systolic reservoir phase and the diastolic passive (conduit) and active emptying (booster pump) phases. To characterize these functions, stroke volumes (SVs) and emptying fractions (EFs) were calculated from the above-mentioned volumes as follows:

\section{Left atrial stroke volumes}

- Total Atrial Stroke Volume (TASV): $\mathrm{V}_{\max }-\mathrm{V}_{\min }$ (reservoir function)

- Passive Atrial Stroke Volume (PASV): $\mathrm{V}_{\max }-\mathrm{V}_{\text {preA }}$ (conduit function)

- Active Atrial Stroke Volume (AASV): $V_{\text {pre }} A^{-} V_{\text {min }}$ (booster pump/active contraction function) 


\section{Left atrial emptying fractions}

- Total Atrial Emptying Fraction (TAEF): TASV/ $\mathrm{V}_{\max } \times 100$ (reservoir function)

- Passive Atrial Emptying Fraction (PAEF): PASV/V $\max \times 100$ (conduit function)

- Active Atrial Emptying Fraction (AAEF): AASV/V $\mathrm{preA}^{\times 100}$ (booster pump/active contraction function)

\section{DSTE for left atrial strain measurements}

From the same 3D echocardiographic datasets, time curves of unidirectional radial (RS), longitudinal (LS), and circumferential strain (CS) were also generated for each segment using the 16-segment model obtained for LV [81-84] Moreover, due to the ability of 3DSTE to calculate complex strains, area strain (the ratio of endocardial area change during cardiac cycle) and 3D strain (strain in the direction of wall thickening; combination of "unidirectional" strains) were also measured. At each time, segmental strain curve peak strains representing characteristics of reservoir phase of the LA function were measured. Global strains were calculated by the software considering the whole LA while mean segmental strains were obtained as the average of strains of 16 segments as well. These parameters were calculated automatically by the software.

\subsubsection{RESULTS}

COMPARISON OF LEFT ATRIAL VOLUMETRIC, VOLUME-BASED FUNCTIONAL, AND STRAIN PARAMETERS OBTAINED BY THREE-DIMENSIONAL SPECKLE-TRACKING ECHOCARDIOGRAPHY, A NOVEL, NON-INVASIVE CLINICAL TOOL FOR VOLUMETRIC AND STRAIN ANALYSIS, BETWEEN PATIENTS WITH HYPEREOSINOPHILIC SYNDROME AND MATCHED CONTROLS (Aim III.a)

On routine haematological testing, the following results (HES vs. controls) were obtained: RBC: $4.2 \pm 0.5$ T/L vs. $4.3 \pm 0.4 \mathrm{~T} / \mathrm{L}(\mathrm{p}=0.94)$, haemoglobin: $126.7 \pm 18.8 \mathrm{~g} / \mathrm{L}$ vs. $130.1 \pm 10.2 \mathrm{~g} / \mathrm{L}(\mathrm{p}=0.86)$, PLT: $276.3 \pm 176.7 \mathrm{G} / \mathrm{L}$ vs. $282.4 \pm 158.2 \mathrm{G} / \mathrm{L}$, Htc: $36.9 \pm 5.5 \%$ vs. $37.8 \pm 4.9 \%$, WBC: $16.7 \pm 5.8 \mathrm{G} / \mathrm{L}$ vs. $6.8 \pm 1.2 \mathrm{G} / \mathrm{L} \quad(\mathrm{p}=0.02)$, eosinophil ratio: $49.0 \pm 16.6 \%$ vs. $3.2 \pm 2.3 \%(p=0.001)$, and AEC: $8.7 \pm 4.8 \mathrm{G} / \mathrm{L}$ vs. $0.4 \pm 0.1 \mathrm{G} / \mathrm{L}$ ( $\mathrm{p}=0.001)$. No correlation was found between any of the laboratory findings and 2D echocardiographic or 3DSTE data in this patient population. None of the control and HES patients exhibited $>$ Grade 1 mitral or tricuspid regurgitation. Significant difference was only found in LA diameter and interventricular septum thickness between HES and control subjects. Clinical data of each patient with organic involvement are presented in Table 18. Only one patient had a prior cardiac disease (NSTEMI) in his history. 
Table 18. Clinical data of patients with hypereosinophilic syndrome

\begin{tabular}{|c|c|c|c|}
\hline $\begin{array}{c}\text { CASE No. } \\
\text { AGE/SEX/DATE } \\
\text { OF DIAGNOSIS }\end{array}$ & ORGANIC INVOLVEMENT & $\begin{array}{l}\text { HEPATOMEGALY, } \\
\text { SPLENOMEGALY }\end{array}$ & $\begin{array}{c}\text { CARDIAC DISEASE } \\
\text { (YEAR) }\end{array}$ \\
\hline $\begin{array}{c}\text { Case 1 } \\
\text { 52/M/2013 }\end{array}$ & duodenal eosinophilia & splenomegaly & - \\
\hline $\begin{array}{c}\text { Case } 2 \\
71 / \mathbf{M} / 2009\end{array}$ & - & - & - \\
\hline $\begin{array}{c}\text { Case } 3 \\
44 / M / 2010\end{array}$ & - & - & - \\
\hline $\begin{array}{c}\text { Case } 4 \\
66 / F / 2011\end{array}$ & tissue eosinophilia & - & - \\
\hline $\begin{array}{c}\text { Case } 5 \\
77 / \mathbf{M} / 2013\end{array}$ & - & - & NSTEMI (2013) \\
\hline $\begin{array}{c}\text { Case } 6 \\
\text { 69/F/2009 }\end{array}$ & eosinophil dermatitis & - & - \\
\hline $\begin{array}{c}\text { Case } 7 \\
45 / M / 2011\end{array}$ & $\begin{array}{l}\text { sensoral motoneuritis, pulmonal } \\
\text { affection, sural necrotising } \\
\text { granulomatous vasculitis }\end{array}$ & splenomegaly & - \\
\hline $\begin{array}{c}\text { Case } 8 \\
\text { 59/M/2013 }\end{array}$ & - & - & - \\
\hline $\begin{array}{c}\text { Case } 9 \\
41 / \mathrm{F} / 2002 \\
\end{array}$ & 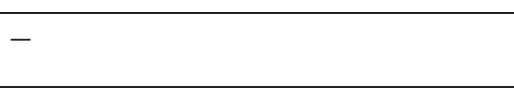 & - & - \\
\hline $\begin{array}{c}\text { Case } 10 \\
73 / \mathbf{F} / 2014\end{array}$ & bronchial asthma, vasculitis & - & - \\
\hline
\end{tabular}

NSTEMI, non-ST-segment elevation myocardial infarction

As reflected by 3DSTE data, both global and mean segmental peak CS were significantly reduced in HES patients suggesting an impaired LA reservoir function (Table 19).

Table 19. Comparison of 3DSTE-derived global and mean peak segmental strain parameters of patients with hypereosinophilic syndrome and controls

\begin{tabular}{|lccc|}
\hline & $\begin{array}{c}\text { HES patients } \\
(\mathrm{N}=10)\end{array}$ & $\begin{array}{c}\text { Controls } \\
(\mathrm{N}=19)\end{array}$ & p-value \\
\hline Global strain parameters & & & \\
$\quad$ Radial strain (\%) & $-17.7 \pm 7.7$ & $-15.7 \pm 11.6$ & 0.64 \\
Circumferential strain (\%) & $18.3 \pm 6.7$ & $25.6 \pm 9.0$ & 0.03 \\
Longitudinal strain (\%) & $21.0 \pm 6.2$ & $22.3 \pm 8.7$ & 0.68 \\
3D strain (\%) & $-10.1 \pm 5.0$ & $-9.3 \pm 9.0$ & 0.81 \\
Area strain (\%) & $41.2 \pm 13.8$ & $50.7 \pm 20.4$ & 0.20 \\
Mean segmental strain parameters & & & \\
Radial strain (\%) & $-20.6 \pm 6.1$ & $-19.5 \pm 8.1$ & 0.70 \\
Circumferential strain (\%) & $22.2 \pm 6.0$ & $31.0 \pm 12.1$ & 0.04 \\
Longitudinal strain (\%) & $21.8 \pm 6.4$ & $25.6 \pm 7.5$ & 0.18 \\
3D strain (\%) & $-14.7 \pm 4.3$ & $-13.7 \pm 6.7$ & 0.67 \\
Area strain (\%) & $45.6 \pm 13.1$ & $58.3 \pm 21.7$ & 0.10 \\
\hline
\end{tabular}

3D, three-dimensional; HES, hypereosinophilic syndrome. 
Significantly increased maximum $(\mathrm{p}=0.01)$ and minimum $(\mathrm{p}=0.03)$ LA volumes as well as LA volume before atrial contraction $(p=0.01)$ and elevated total $(p=0.02)$ and active $(p=0.005)$ atrial SVs values characterizing reservoir and booster pump LA function were found in HES patients, as compared to controls. EF did not significantly differ between groups (Table 20).

Table 20. Comparison of 3DSTE-derived volumetric and volume-based functional left atrial parameters of patients with hypereosinophilic syndrome and controls

\begin{tabular}{|c|c|c|c|}
\hline & $\begin{array}{l}\text { HES patients } \\
\qquad(\mathrm{N}=10)\end{array}$ & $\begin{array}{l}\text { Controls } \\
(\mathrm{N}=19)\end{array}$ & p-value \\
\hline \multicolumn{4}{|l|}{ Calculated volumes } \\
\hline Maximum LA volume $\left(\mathrm{V}_{\max }, \mathrm{mL}\right)$ & $72.9 \pm 38.8$ & $45.6 \pm 15.5$ & 0.01 \\
\hline $\mathrm{V}_{\max } / \mathrm{BSA}\left(\mathrm{mL} / \mathrm{m}^{2}\right)$ & $41.8 \pm 25.0$ & $26.0 \pm 9.7$ & 0.03 \\
\hline $\mathrm{V}_{\max } / \mathrm{BMI}\left[\mathrm{mL} /\left(\mathrm{kg} / \mathrm{m}^{2}\right)\right]$ & $2.8 \pm 1.5$ & $1.8 \pm 0.6$ & 0.03 \\
\hline Minimum LA volume $\left(\mathrm{V}_{\min }, \mathrm{mL}\right)$ & $46.3 \pm 33.3$ & $26.0 \pm 15.0$ & 0.03 \\
\hline $\mathrm{V}_{\min } / \mathrm{BSA}\left(\mathrm{mL} / \mathrm{m}^{2}\right)$ & $26.8 \pm 21.4$ & $14.9 \pm 9.7$ & 0.05 \\
\hline $\mathrm{V}_{\min } / \mathrm{BMI}\left[\mathrm{mL} /\left(\mathrm{kg} / \mathrm{m}^{2}\right)\right]$ & $1.7 \pm 1.2$ & $1.0 \pm 0.6$ & 0.05 \\
\hline $\begin{array}{l}\text { LA volume before atrial contraction }\left(\mathrm{V}_{\text {preA, }} \text {, }\right. \\
\mathrm{mL})\end{array}$ & $62.0 \pm 36.0$ & $36.5 \pm 16.6$ & 0.01 \\
\hline $\mathrm{V}_{\mathrm{preA}} / \mathrm{BSA}\left(\mathrm{mL} / \mathrm{m}^{2}\right)$ & $35.7 \pm 23.1$ & $20.9 \pm 10.4$ & 0.03 \\
\hline $\mathrm{V}_{\text {preA }} / \mathrm{BMI}\left[\mathrm{mL} /\left(\mathrm{kg} / \mathrm{m}^{2}\right)\right]$ & $2.3 \pm 1.4$ & $1.4 \pm 0.7$ & 0.03 \\
\hline \multicolumn{4}{|l|}{ Stroke volumes (SVs) } \\
\hline Total atrial SV (TASV, mL) & $26.6 \pm 8.5$ & $19.6 \pm 6.4$ & 0.02 \\
\hline TASV / BSA (mL/m²) & $15.0 \pm 4.9$ & $11.1 \pm 3.6$ & 0.04 \\
\hline TASV / BMI $\left[\mathrm{mL} /\left(\mathrm{kg} / \mathrm{m}^{2}\right)\right]$ & $1.0 \pm 0.3$ & $0.8 \pm 0.3$ & 0.12 \\
\hline Passive atrial SV (PASV, mL) & $10.9 \pm 8.2$ & $9.1 \pm 5.0$ & 0.47 \\
\hline $\mathrm{PASV} / \mathrm{BSA}\left(\mathrm{mL} / \mathrm{m}^{2}\right)$ & $6.1 \pm 4.6$ & $5.1 \pm 2.8$ & 0.61 \\
\hline PASV / BMI [mL/(kg/m²)] & $0.4 \pm 0.3$ & $0.4 \pm 0.2$ & 0.78 \\
\hline Active atrial SV (AASV, mL) & $15.7 \pm 5.1$ & $10.5 \pm 4.0$ & 0.005 \\
\hline $\mathrm{AASV} / \mathrm{BSA}\left(\mathrm{mL} / \mathrm{m}^{2}\right)$ & $8.9 \pm 2.9$ & $5.9 \pm 2.3$ & 0.01 \\
\hline $\mathrm{AASV} / \mathrm{BMI}\left[\mathrm{kg} /\left(\mathrm{mL} / \mathrm{m}^{2}\right)\right]$ & $0.6 \pm 0.2$ & $0.4 \pm 0.2$ & 0.03 \\
\hline \multicolumn{4}{|l|}{ Emptying fractions (EFs) (\%) } \\
\hline Total atrial EF & $40.0 \pm 10.5$ & $45.0 \pm 12.9$ & 0.29 \\
\hline Passive atrial EF & $15.9 \pm 11.7$ & $21.4 \pm 10.8$ & 0.21 \\
\hline Active atrial EF & $28.6 \pm 7.8$ & $30.5 \pm 9.5$ & 0.58 \\
\hline
\end{tabular}

BMI, body mass index; BSA, body surface area; HES, hypereosinophilic syndrome; LA, left atrial. 
PRESENTATION OF LEFT VENTRICULAR ROTATIONAL MECHANICS THROUGH A UNIQUE CASE OF HYPEREOSINOPHILIC SYNDROME WITH LOEFFLER'S ENDOCARDITIS BY MEANS OF THE NOVEL METHOD OF 3DSTE (Aim III.b) [85]

Loeffler's disease is associated with stiffened ventricular and atrial walls leading to inadequate filling, decreased preload, diastolic dysfunction, and heart failure. At this moment, little is known about the rotational characteristics of the Loeffler's heart. Therefore, we present a 36-year-old male patient with known Loeffler's endocarditis on optimal therapy whose rotational parameters were evaluated by 3DSTE. The multiple long- (A, B) and shortaxis views $(\mathrm{C} 3, \mathrm{C} 5, \mathrm{C} 7)$ extracted from a 3D echocardiographic dataset are shown in Figure 6. Visual information on LV rotation is given in colour overlay superimposed on grey-scale images. Moreover, a 3D cast of LV (Figure 6D) and calculated LV volumes, ejection fraction (EF), and estimated LV mass are also demonstrated (Figure 6E). Quantitative data are provided on LV rotation with adequate rotational directions with counterclockwise motion of the LV apex (white arrow, positive value) and clockwise motion of the LV base (dashed arrow, negative value) (Figure 6F). Both LV apical and basal rotation were in normal range suggesting normal rotational characteristics despite reduced systolic function in this case with known Loeffler's disease. Data of a control subject are also presented in the same fashion (Figure 7) [85].

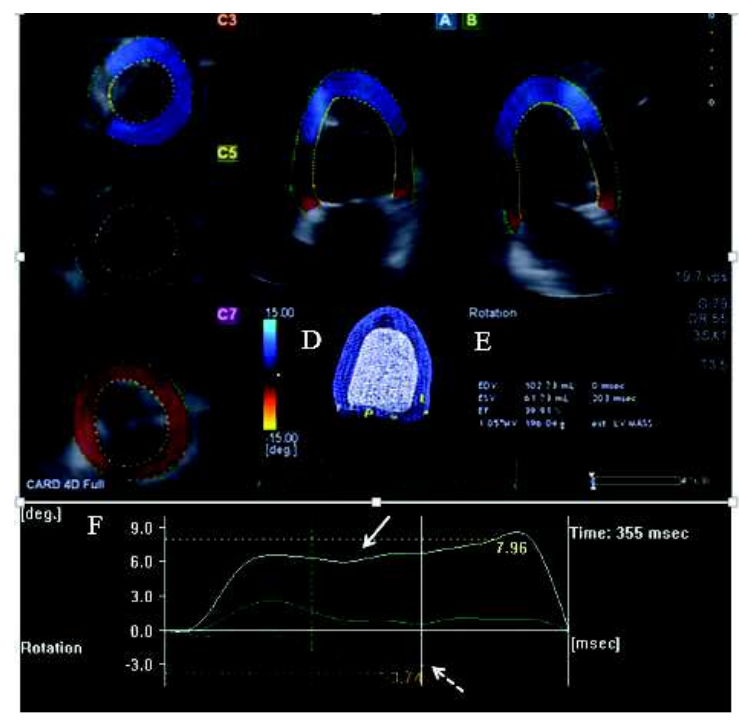

Figure 6. Apical four-chamber (A) and twochamber (B) views and short-axis views (C3, C5, C7) at different levels of the left ventricle (LV) extracted from the three-dimensional (3D) echocardiographic dataset. A $3 D$ cast of the $L V(D)$ and calculated volumetric and functional $L V$ parameters (EDV, end-diastolic volume; ESV, endsystolic volume; EF, ejection fraction; est LV MASS, estimated left ventricular mass) are also demonstrated (E). Counterclockwise rotation of $L V$ apex (white arrow, positive value) and clockwise rotation of LV base (dashed arrow, negative value) are also shown demonstrating normal rotational directions in this Loeffler patient's heart $(F)$. 


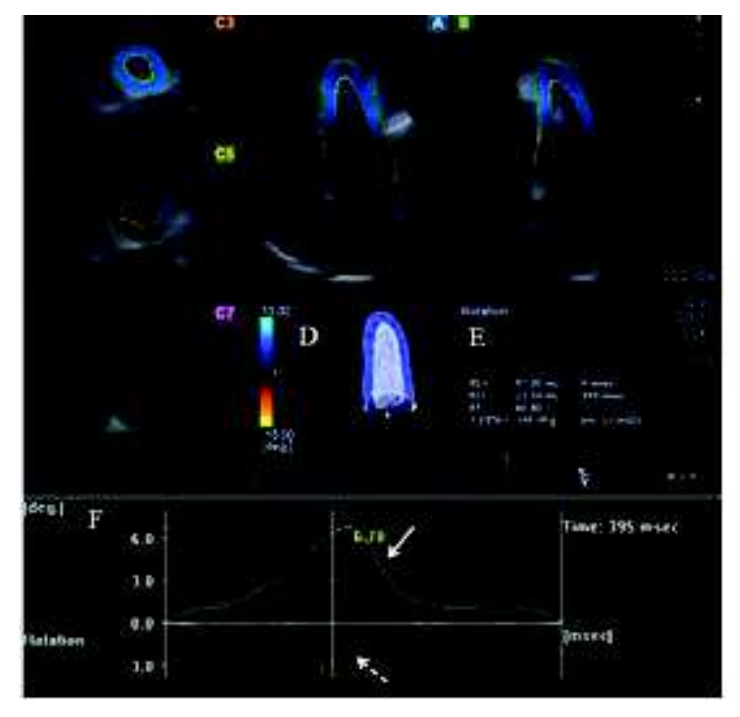

Figure 7. Apical four-chamber (A) and twochamber (B) views and short-axis views (C3, C5, C7) at different levels of the left ventricle (LV) extracted from the three-dimensional (3D) echocardiographic dataset. A $3 D$ cast of the $L V(D)$ and calculated volumetric and functional $L V$ parameters (EDV, enddiastolic volume; ESV, end-systolic volume; EF, ejection fraction; est LV MASS, estimated left ventricular mass) are also demonstrated (E). Counterclockwise rotation of $L V$ apex (white arrow, positive value) and clockwise rotation of $L V$ base (dashed arrow, negative value) are also shown demonstrating normal rotational directions in a healthy subject $(F)$.

Moreover, it is important to emphasize that our research identified an uncommon case in the investigated patient population. Due to its unique nature and potential clinical relevance, we demonstrated it in a separate case report, providing therefore an additional proof of the complexity of HES [86]. The case is presented in details in Appendix VI to this dissertation as the publication of Imelda Marton et al.: Watershed infarction in hypereosinophilic syndrome: a diagnostic dilemma in FIP1L1-PDGFR-alpha-associated myeloid neoplasm and overview of the relevant literature [86].

\section{DISCUSSION}

\subsection{POLYCYTHAEMIA VERA}

$\mathrm{PV}$, the most common $B C R-A B L 1$-negative classic MPN is a HSC-derived clonal myeloproliferation characterized by a trilineage expansion of morphologically normal red cells, white cells, and platelets without significant bone marrow fibrosis. At the $2^{\text {nd }}$ Department of Internal Medicine and Cardiology Centre, which is a regional haematological centre for the population of south-eastern Hungary, data on 108 PV patients were available from the period between 1998 and 2014. As reported [10], the incidence of PV varies from 0.4 to 2.8 cases per 100,000 persons per year in the European Union; the median age at diagnosis is around 61 years (range: 18-95 years) [11]. The occurrence of PV is a bit higher in men than in women $(2.8$ vs. 1.3 cases per 100,000 persons per year [12]. In our 
study, demographics like distribution by gender and mean age at diagnosis were highly consistent with corresponding data in the literature.

The diagnostic criteria of PV have been changed several times since the first edition of the PVSG criteria in 1975. The identification of $J A K$ mutation fundamentally affected the diagnosis of PV. The acquired JAK2 V617F somatic mutation causes cytokine-independent activation of several biochemical pathways involved in the erythropoietin receptor signalling. Given that more than $90 \%$ of PV patients carry this particular mutation, it has been incorporated into the 2008 WHO diagnostic criteria. The lack of clear diagnostic criteria in the past often resulted a proportion of secondary polyglobulia cases to be diagnosed and followed as PV. Therefore, we strictly adhered to the 2008 WHO criteria while establishing our PV database and excluded all polyglobulia cases which did not meet these criteria (i.e., cases without $J A K 2$ mutation, combined with normal or increased serum erythropoietin levels). The proportion of PV patients with JAK2 V617F mutation in our PV database was $94.4 \%$ which correlates well with the existing literature [17]. The incidence of $J A K 2$ exon 12 mutation (which has a reported frequency of 3\%) was not investigated in our research. While earlier studies estimated the rate of vascular events to $12-39 \%$ and reported about various manifestation forms [18], recent works have also confirmed their incidence well above $20 \%$ [17]. Arterial events occur typically much more frequent than venous ones [20].

In PV, thrombosis (splanchnic venous thrombosis or cerebral sinus thrombosis) is often an initial occurrence which may suggest the diagnosis as early as before the haematological verification. In our study, the prediagnostic incidence of major thrombotic events was 33/108 (30,5\%), highly similar to literature data [87]. Another research found the frequency of pre- and peridiagnostic major vascular events as $16 \%$ for arterial and $7 \%$ for venous complications, respectively, while the incidence of haemorrhagic events made up 4\% [88]. In the largest prospective European PV study (European Collaboration Study on Lowdose Aspirin in Polycythemia - ECLAP, 2004), the same incidence rates accounted for 27\%, $11 \%$, and $9.2 \%$, respectively [89]. Later investigations like the CYTO-PV trial (2013) reported $17 \%$ and $12 \%$ for the incidence of arterial and venous complications, respectively, while these numbers were $12 \%$ and $9 \%$ in another study conducted in 1545 patients by Tefferi et al. (2013) [11, 88, 90]. Decreasing incidence rates might be explained by the evolution of diagnostic criteria and the incorporation of novel knowledge into the therapeutic regimens (e.g., based on results from the ECLAP study, wider use of ASA, more aggressive phlebotomy policy for more restrictive Hct target, introduction of cytoreductive treatment, and more intensive management of $\mathrm{CV}$ risk factors). 
In our PV patient cohort representing the period from 1998 to 2014, a total of 20 major vascular complications were observed in 108 patients (18.5\%); among them, arterial events $(\mathrm{n}=18 ; 16.6 \%)$ occurred more frequently then venous ones $(\mathrm{n}=2 ; 1.8 \%)$. The frequency of total vascular and arterial events in our research was similar to those in the above trials, with consistently lower rates for venous thrombotic events. Furthermore, we thoroughly investigated the incidence of $\mathrm{CV}$ events during follow-up and found them to occur in 7 cases in 108 patients $(6 \%)$. This means, six out of seven PV patients were available for an adequate cardiological analysis of $\mathrm{CV}$ complications during follow-up. All patients belonged to the high-risk group and five of them (83\%) had at least two or more conventional CV risk factors. The frequency of AMI in our PV cohort was higher (6\%) than that reported in trials on larger patient populations $(2 \%)$ [88].

Compared to the literature (7\%), we detected a markedly higher frequency for cerebrovascular events $(11 / 108 ; 10.1 \%)$ [88]. The occurrence of cerebrovascular complications in our PV cohort also exceeded the incidence of stroke reported earlier in the general Hungarian population $(2 / 1,000$ in the age of $45-54$ years, $3 / 1,000$ in the age of 5564 years, and 3-13/1,000 above the age of 65 years; 2005) [91]. In most cases, chronic ischaemic white matter lesions were observed. Mild cerebral atrophy was also a frequent finding. The clinical presentation of cerebrovascular events was predominated by lacunar syndromes or VBI. Most of these patients $(8 / 11 ; 72 \%)$ also presented at least two major conventional CV risk factors. The slightly higher AMI and stroke incidence rates in our PV cohort might be attributed to our less stringent - yet historically guideline-compliant - attitude in terms of Hct targets and the introduction of cytoreductive treatment at that time; i.e., our treatment strategy used to be based on the conventional two-level risk assessment. In addition, the significant additional effect of $\mathrm{CV}$ risk factors on thrombotic events was only partially acknowledged and this approach in PV management was therefore much less dominant.

Although CV risk factors were incorporated into the IPSET score in 2012 for purposes of thrombosis risk stratification in ET, the same practice is still disputed for PV [92]. The results of our research in PV in terms of the incidence of $\mathrm{CV}$ and cerebrovascular complications and the contribution of $\mathrm{CV}$ risk factors to subsequent thrombotic complications clearly demonstrate the importance of considering PV as a prothrombotic state where conventional CV risk factors (hypertension, tobacco use, hyperlipidaemia, obesity, DM) may significantly increase the risk of thrombotic events. The complete pathomechanism of prothrombotic processes in PV remains, however, to be understood. Falanga et al. carried out intensive investigations on the correlation between MPNs and thromboses and found the 
pathogenesis to be particularly complex and multifactorial. The higher thrombosis risk in PV can be considered as a result of as a consequence of an acquired thrombophilic state associated with the underlying disease. This might be partly explained by the prothrombotic characteristics of MPN clone-derived blood cells and the procoagulant response of normal epithel cells to inflammatory stimuli. In addition, higher Hct rates result in blood hyperviscosity. The greater red cell mass displaces platelets toward the vessel wall, thus facilitating shear-induced platelet activation and aggregation and enhancing platelet-platelet interaction. Moreover, changes of the red cell membrane trigger the formation of erythrocyte aggregates. Finally, all these processes together are responsible for the increased coagulability of blood in PV [20]. Although the recommended Hct level was questioned for a long time, [93], current gudelines clearly indicate a target of 45\% in PV [17, 21, 90]. Our observation that the frequency of thrombotic events during follow-up differed significantly between PV subgroups with Hct values below or above $45 \%$ is well reflected by the latest therapeutic recommendations.

Currently, no data are available whether the presence of one CV risk factor or two or more CV risk factors considerably increases the occurrence of thrombotic complications in PV. Our research demonstrated that there is a significant difference in the thrombosis-free survival between PV patients with or without at least one CV risk factor. The difference is also significant for the comparison of PV patients with at most one CV risk factor or at least two CV risk factors. These results were further confirmed by our study to evaluate the role of $\mathrm{CV}$ risk factors in major thrombotic complications in the pooled population of female patients with ET or PV. In this setting, the presence of two or more CV risk factors was associated with a significantly higher risk of thrombosis. Moreover, a significant difference was seen in the thrombosis-free survival between patients with at most one CV risk factor and those with two or more CV risk factors [25]. Female patients with CV risk factors and PV or ET may well be at a higher risk of thrombotic events, and require therefore a special consideration for the prevention and management of thrombotic events.

Regarding thrombotic events, the evaluation of additional risk factors is a novel approach. The first papers emphasizing the role of CV risk factors in addition to conventional risk assessment (age $>60$ years and/or prior thrombotic event) were published in the last year of our research. Moreover, the 2015 update on PV management by Tefferi et al. implementing new results provides much more complex recommendations than the previous versions used to be based on a two-level (low or high) risk assessment [17]. Although CV risk factors were not incorporated in the risk stratification of the 2015 guidelines of the European Society for 
Medical Oncology, they are taken into account in their risk-adapted therapy recommendations [94]. Given that in PV, thrombohaemorrhagic events represent the leading cause of morbidity and mortality, the therapy primarily aims to prevent these complications without increasing the bleeding risk, and only secondarily to control the symptoms. Therefore, treatment should always be individually adjusted to thrombosis and/or bleeding risk. Knowledge is being continuously updated and implemented in the guidelines, resulting in even more stringent therapeutic recommendations. Controlled trials showed the reduction of thrombosis risk with low-dose ASA and cytoreductive therapy in high-risk patients, without affecting the survival $[89,90]$. Our results support the importance of individualized treatment - considering each patient's thrombosis and/or bleeding risk - and more aggressive management of modifiable risk factors (e.g. CV risk factors) with restrictive targets in PV.

Patients defined as being at low risk according to previous therapeutic guidelines (age $<60$ years, without prior thrombotic event) used to be treated with anti-platelet agents (e.g. ASA) and by phlebotomy, while high-risk patients (age $\geq 60$ years and/or with prior thrombotic event) used to receive cytoreductive drugs (e.g. hydroxyurea) combined with anti-platelet therapy. Current therapeutic recommendations for PV are largely adapted to individual risk profile. For low-risk patients (age $<60$ years and negative thrombosis history and the presence of JAK2 V617F mutation or CV risk factor(s)) without extreme thrombocytosis, low-dose ASA once daily is recommended; in case of CV risk factors with concomitant $J A K 2$ V617F mutation positivity ASA twice daily should be considered. In lowrisk patients with extreme thrombocytosis $\left(\mathrm{PLT}>1,000 \times 10^{9} / \mathrm{L}\right)$, the measurement of ristocetin cofactor activity is necessary (administration of ASA if $>30 \%$ ). High-risk patients (age $\geq 60$ years and/or positive thrombotic history) without prior thrombotic events should be treated with hydroxyurea + once daily low-dose ASA. In case of previous arterial thrombotic event, hydroxyurea + ASA should be given, with the consideration of ASA twice daily if any or more of the following are present (CV risk factor(s), JAK2 V617F mutation positivity, age $>60$ years). For high-risk patients with previous venous event, hydroxyurea with life-long anticoagulation is recommended, given the concept of $\mathrm{PV}$ as an acquired persistent thrombophilic state. The addition of ASA once daily should also be considered if any or more of the following are present ( $\mathrm{CV}$ risk factor(s), JAK2 V617F mutation positivity, age $>60$ years). In all patients, pharmacological therapy should always be completed by phlebotomy to target Hct $<45 \%[17,95]$. For high-risk patients refractory to or intolerant of hydroxyurea, interferon-alpha (IFN-alpha; age $<65$ years) or busulphan (age $>65$ years) may be an option. The selective JAK1/2 inhibitor ruxolitinib approved in 2015 for the treatment of 
adult patients with PV who are resistant to or intolerant of hydroxyurea is not yet widely available in Hungary.

\subsection{SYSTEMIC MASTOCYTOSIS}

Mastocytosis is an orphan disease characterized by the clonal neoplastic proliferation of mast cells accumulating in one or more organ systems. Out of the several manifestations of adult mastocytoses, our research targeted adult SM. According to the $2008 \mathrm{WHO}$ criteria, SM is classified as ISM, SM-AHNMD, ASM, and MCL. In our regional centre, 14 ISM, 15 AHNMD, and 6 ASM cases were diagnosed in the period between 2001 and 2013; MCL was, however, not detected in any patient. As our study aimed at the evaluation of histological features, molecular characteristics as well as laboratory and clinical parameters of adult SM, mastocytosis cases in paediatric patients or those in adults confined only to the skin (without available bone marrow biopsy results) were not included in our research. All bone marrow test results since 2001 were screened for SM and re-assessed by morphological, immunohistochemical, and molecular (PCR) methods, according to the 2008 WHO criteria.

ISM is the most frequent subtype of SM in adults. It is predominated by cutaneous manifestations (UP) but recurrent systemic symptoms related to mast cell degranulation and mediator release and/or allergies or anaphylaxis may also occur. A wide range of factors may lead to mast cell activation, resulting in severe or even life-threatening or fatal symptoms [26, 38]. In our SM study population, cutaneous manifestation (UP) was detected in 57\% (8/14) of the patients while mediator-related symptoms occurred in $28 \%$ (4/14) of them.

In our study, the main cause of the indication of bone marrow biopsy in the group of ISM patients was UP $(8 / 14 ; 57 \%)$. It is important therefore that dermatologists should refer any patient with $\mathrm{CM}$ lesions to a haematological centre to check potential bone marrow involvement. In other cases, bone marrow biopsy was performed for eosinophilia $(3 / 14 ; 21 \%)$, anaemia $(1 / 14 ; 7 \%)$, lytic bone lesions $(1 / 14 ; 7 \%)$, or adenomegaly $(1 / 14 ; 7 \%)$.

On the contrary, symptoms in ASM (e.g. cytopenia, ascites, malabsorption, or osteolytic skeletal lesions) result from organ dysfunction due to mast cell infiltration. In the entire ASM subgroup $(6 / 6 ; 100 \%)$, bone marrow biopsy was requested due to either anaemia with or without thrombocytopenia or hepatosplenomegaly with or without constitutional symptoms [55]. All ASM patients presented with at least one C finding, as defined by the 2008 WHO criteria (marked cytopenia, osteolysis with or without pathologic fractures, ascites and elevated liver enzymes, malabsorption with hypalbuminaemia, palpable splenomegaly with hypersplenism). 
In the SM-AHNMD subgroup, mainly symptoms of the associated neoplasm indicated bone marrow biopsy. In most patients $(14 / 15 ; 93 \%)$, these were symptoms of pancytopenia, anaemia, leukocytosis, thrombocytosis, bone pain/lesion, or hepatosplenomegaly while in one case, the associated SM was revealed by concurrent lymphoma staging process. Regarding associated clonal diseases, our findings were consistent with previous reports [27]. Also in our study, primarily myeloid neoplasms were identified $(12 / 15 ; 80 \%)$ while lymphoid neoplasms were much less frequently detected $(3 / 15 ; 20 \%)$. As provided in current literature [46], we found ISM to be mainly accompanied by cutaneous symptoms $(8 / 14 ; 57 \%)$; however, we observed a lower rate of this association in ASM $(2 / 6 ; 33 \%)$ and the lack of such manifestation in SM-AHNMD.

Most of the adult patients with mastocytosis, regardless of disease subtype, are positive for the somatic activating mutation of the oncogenic receptor tyrosine kinase KIT (exon 17, D816V) [33, 42-45] The KIT D816V mutation, which is found in up to $85 \%$ of all SM patients, is of both pathogenetic and diagnostic relevance [26, 43-45].

In our SM patient population, $C$-KIT mutations detected by PCR were confirmed by Sanger sequencing. Out of all detected cases, only those were enrolled in the SM study which strictly met the relevant 2008 WHO criteria (major criterion and $\geq 1$ minor criterion, OR $\geq 3$ minor criteria). In accordance with literature data, KIT D $816 \mathrm{~V}$ mutation positivity was detected in $78 \%(11 / 14)$ of ISM patients, $80 \%(12 / 15)$ of AHNMD patients, and $83 \%(5 / 6)$ of ASM patients, respectively $[31,96]$.

The clinical presentation of SM is highly heterogeneous ranging from asymptomatic manifestations (ISM) to severe, progressive disease forms greatly affecting life expectancy (ASM, MCL) [31]. The correct diagnosis is therefore of crucial importance, combining the analysis of histopathological and molecular characteristics with the assessment of B and C findings. The complex evaluation of these parameters will determine which WHO subcategory each patient is to be assigned to. The updated 2008 WHO nomenclature with its strong prognostic implications provides a much better prediction of the typical clinical course and the expected survival in each SM subtype. A long-term Spanish study revealed that the great majority of adult ISM patients are able to live a normal life [97].

The results of the ever largest trial to confirm the prognostic value of the current WHO classification system were published in 2009 [27]. Within this study, clinical, laboratory, and survival data of 342 adult patients diagnosed with SM over a 30-year-long period were recorded and evaluated. Compared to the non-indolent forms of SM (AHNMD and ASM), the life expectancy in the ISM group was considerably higher and not significantly different from 
the age- and sex-matched American (USA) population for the appropriate time period, based on the date of diagnosis [27].

The aim of our research was to analyse the set of adult SM cases emerged in a cohesive geographic region of Hungary. The distribution of SM subtypes in our study population of 35 patients was mostly similar to that reported in the largest clinical trial in SM with the updated WHO criteria: ISM 14/35 (40\%), SM-AHNMD 15/35 (42\%), ASM 6/35 (17\%) and MCL $0 \%$, vs. $46 \%, 40 \%, 12 \%$, and $1 \%$, respectively $[27,50]$.

Our analyses on Hungarian patients revealed that the survival in SM (including all subtypes) is worse than that expected in the age- and sex-matched Hungarian population. The life expectancy of patients with ISM was excellent whereas SM-AHNMD and ASM groups had a reduced median survival. The distribution of subtypes and the survival pattern seen in our SM study population was similar to that previously reported in the Mayo Clinic trial $[27,50]$.

To date, only limited epidemiologic data are available in SM. The prevalence of mastocytosis in Central Europe is estimated at 0.5-1/10,000 [37]. Local/regional data on ISM have been reported from The Netherlands (Groningen) and population-based epidemiological data from Denmark [36, 38, 39]. Moreover, the recently established centralized ECNM registry is still collecting data from ten European countries; therefore, epidemiological data on the prevalence of SM are already available [36]. We found the 13-year cumulative incidence of SM in the general population aged 15 years or more amounts to $0.27 / 10,000$ which meets the criterion of orphan diseases. As in the most relevant literature [37], we could not report a gender predominance. Our data on the cumulative incidence of SM are the first such results published in Hungary. Since only cases that strictly complied with the WHO criteria were included in our SM investigations, the cumulative incidence of SM calculated during our research is likely to be somewhat underestimated. Patients with skin lesions who refused bone marrow biopsy were not enrolled in our analyses. The real cumulative incidence might be higher because SM is often underdiagnosed due to its subtle or even absent symptoms.

Within the SM cohort, we individually reported the course of a female patient with ISM followed-up for 27 years, an unusual case of particular clinical relevance [54]. During this uniquely long follow-up, she experienced recurrent specific cutaneous symptoms which greatly impaired her quality of life. As her cutaneous symptomes showed only slight and temporary improvement on multiple symptomatic treatments (antihistamine, sodium cromoglicate, psoralen + ultraviolet A light [PUVA] therapy, IFN-alpha), imatinib mesylate was introduced even though neither imatinib-resistant KIT D $816 \mathrm{~V}$ nor any imatinib-sensitive KIT mutation was identified. Unexpectedly, the patient with this KIT D816V mutation- 
negative disease achieved a considerable reduction of skin lesions and a temporary appreciable improvement of her quality of life on imatinib.

Currently, there is no curative treatment for SM. The available therapies (including histamine receptor antagonists and other antimediator agents) give only symptomatic relief by decreasing the effects of mast cell activation. An important component of SM treatment is the elimination of known symptom triggers. The perioperative management of SM patients is particularly difficult: a multidisciplinary preoperative assessment, an adequate premedication, and a close intra- and postoperative monitoring are of outstanding importantance [28]. Cytoreductive and targeted therapies (tyrosine-kinase inhibitors) can only be considered in aggressive and leukaemic SM variants. As the vast majority of SM cases harbour the known imatinib-resistant KIT D816V mutation, currently available tyrosine-kinase inhibitors are, unfortunately, ineffective. Other drugs like multikinase inhibitors are still under clinical investigation. The prognosis of these patients remains poor, even if treated with novel Kittargeting agents, polychemotherapy, or HSC transplantation [26].

\subsection{HYPEREOSINOPHILIC SYNDROME}

Cardiac manifestations are the major cause of morbidity in HES and develop in three stages. The first acute, mostly asymptomatic necrotic stage is due to eosinophilic infiltration of the myocardium. The initial damage is thought to be mediated by the contents of the eosinophilic granules. The intermediate (thrombotic) phase is characterized by thrombus formation followed by thrombus organization into a thick layer of granulation tissue. In the third, fibrotic stage, granulation tissue evolves into fibrosis with a small inflammatory zone. Nowadays, the term "Loeffler's endomyocarditis" is used to describe the thrombotic and fibrotic stage of cardiac involvement in HES. Typical echocardiographic findings include endocardial thickening, fibrothrombotic obliteration of the ventricular apices, and valvular regurgitation due to restricted motion of the posterior mitral leaflet as assessed by routine $2 \mathrm{D}$ Doppler echocardiography. At enrolment, the majority of our HES patients did not have any known cardiovascular disease (except for Case 5) or clinical signs of thrombosis/fibrosis characteristic of Loeffler's endocarditis. They represented theoretically the first asymptomatic - stage of the disease; therefore, any subsequent alteration in LA morphology and function could be attributed solely to HES. Only LV hypertrophy and dilated LA could be detected by conventional 2D Doppler echocardiography without significant valvular regurgitations or thrombus formation. 3DSTE confirmed LA volumetric changes in all phases of LA function and found alterations in both global and mean peak segmental LA-CS, 
suggesting a reduced LA reservoir function and remodelling. Wide spectrum of pathophysiological changes could lead to LA remodelling with structural, functional, or neurohormonal etc. consequences. The real mechanism behind LA remodelling in HES is not completely known, but myocyte necrosis, alterations of the extracellular matrix and in the release of atrial hormones due to toxic proteins from degranulating eosinophils, and diastolic dysfunction could explain our findings.

3DSTE is a new clinical tool for non-invasive 3D cardiac chamber quantification of the LV and LA. The technique is based on the so-called "block-matching algorithm" of the myocardial speckles during their frame-to-frame motion. 3DSTE has been demonstrated to be useful for LA volumetric and strain assessments, allowing more detailed evaluation of LA function from the same 3D dataset. Different patterns in 3DSTE-derived volume-based and strain functional properties could be demonstrated in different disorders. In a recent 3DSTE study, peak LA-RS and LA-LS were found to be altered in hypertrophic cardiomyopathy along with preserved LA-CS. In another study, all strains at all LA levels showed alterations in atrial fibrillation by 3DSTE. In the present study, only peak LA-CS was decreased while RS and LS remained unchanged in HES patients. The pathomechanism of this phenomenon, i.e. only LA-CS showed alterations in HES is unknown, but haemodynamic factors and their relationship with LA fiber orientation could not be excluded, in addition to the above processes [7-9, 79-81].

Our investigations were the first in HES with 3DSTE. Moreover, it is important to emphasize that our research identified an uncommon case. Due to its unique nature and potential clinical relevance, we demonstrated it in a separate case report, providing therefore an additional proof of the complexity of HES. The patient with hyperosinophilia in our report [86] had involvement of the CNS and the myocardium. After the onset of neuropsychiatric symptoms, magnetic resonance imaging indicated bilateral cerebral and cerebellar corticalsubcortical lesions involving the watershed areas, mainly in the parieto-occipital regions. The first transthoracal echocardiography (TTE) during the presence of neurological symptoms did not reveal any pathological findings, but repeated TTE two weeks later suggested the involvement of the myocardium in the form of Loeffler's endocarditis. Abdominal ultrasonography showed splenomegaly. Clinical data and bone marrow histopathology confirmed the diagnosis of HES. No chromosomal aberration was detected, and subsequent molecular tests by FISH demonstrated FIPIL1-PDGFRA gene rearrangement. High-dose intravenous steroid (methylprednisolone $500 \mathrm{mg}$ /day) alleviated the neurological symptoms within a few weeks while low-dose imatinib (200 mg/day) resulted in an impressive 
regression of hypereosinophilia and splenomegaly in 6 weeks. The pathogenesis of the neurological dysfunction in HES is potentially explained by a number of hypotheses: a) direct infiltration of eosinophil cells; b) neurotoxic effect of major basic protein and eosinophil cationic protein released from eosinophilic granules; c) local thrombosis due to eosinophilinduced endothelial dysfunction; or d) brain infarction caused by microembolization from endomyocardial fibrosis [98-100]. During follow-up, the patient continued to receive imatinib and experienced persistent remission in a stable condition without developing any new complaints. The diagnostic work-up requires a close and effective multidisciplinary co-operation between the neurologist, the neuroradiologist, cardiologist and the haematologist in order to achieve an early and accurate diagnosis and the successful management of a patient with a FIPIL1-PDGFRA-positive myeloid neoplasm.

For patients with strictly defined HES (i.e., after the exclusion of any possible cause of a secondary hypereosinophilia), basic therapy consists of corticosteroids, with or without hydroxyurea, for the rapid reduction of AEC. For steroid non-responders, hydroxyurea may be administered alone. IFN-alpha can induce both haematological and cytogenetic remission in HES and CEL patients refractory to prior therapies. Currently, anti-interleukin-5 or anti-CD52 monoclonal antibody treatment is mainly used as experimental therapy in patients with PDGFRA/B-negative HES. Treatment choice may vary substantially depending on the underlying eosinophilic condition (whether a targeted molecular therapy is considered or not) and the extent of organ damage.

\section{SUMMARY}

\section{$\underline{\text { CONCLUSIONS AND CLINICAL IMPLICATIONS OF OUR RESEARCH }}$}

For purposes of our research focussing especially on Ph-negative MPNs, we retrospectively established a database of PV, SM, and HES cases diagnosed at the $2^{\text {nd }}$ Department of Internal Medicine and Cardiology Centre between 1998 and 2014. The collected data were analyzed and assessed by disease-relevant factors and in accordance with literature requirements.

\subsection{POLYCYTHAEMIA VERA}

In PV, the most common type of Ph-negative MPDs our research focussed on vascular complications. Vascular events represent the primary cause of morbidity and mortality in PV and require therefore a strong multidisciplinary approach in their diagnosis and treatment. 
- The number of major thrombotic events in PV patients that occurred prior to $(30.32 \%)$ or during the follow-up (14.83\%) was evaluated retrospectively.

- We conducted a separate analysis and evaluation of each and every CV and cerebrovascular event which are usually presented as aggregate data in large-scale trials.

- Regarding cerebrovascular complications in PV, the majority of cerebrovascular complications were chronic ischaemic white matter lesions in our cohort of PV patients. Mild cerebral atrophy was also a frequent finding. The clinical presentation of cerebrovascular events was predominated by lacunar syndromes or VBI. Most of the patients presented at least two serious conventional vascular risk factors, which were supposed to have an impact on both the clinical course of the disease and the morphological alterations seen on brain imaging. These findings suggest that PV predisposes to small vessel cerebral disease manifested primarily as lacunar syndromes, despite the simultaneous presence of additional vascular risk factors in most patients.

- $\quad$ Regarding CV complications, NSTEMI was observed. Most patients had at least two conventional vascular risk factors. Our findings led us to suppose that the early diagnosis followed by percutaneous coronary intervention and an aggressive and personalized management of $\mathrm{CV}$ risk factors may be effective in the prevention of subsequent vascular events. The importance of a close co-operation between the haematologist and specialists in the field of vascular medicine is emphasized.

- $\quad$ The contribution of CV risk factors as newly hypothesized additional risk factors to subsequent thrombotic complications in PV was demonstrated in our patient population. Our findings clearly indicate the importance of the identification and consideration of these risk factors in a more accurate and individualized risk-guided thrombosis management in PV.

\subsection{SYSTEMIC MASTOCYTOSIS}

Analyses in our SM patient group were driven by the orphan nature and the diagnostic, therapeutic, and prognostic difficulties usually seen in this condition. The multidisciplinary significance of SM lies in its challenging diagnostic aspects and characteristic multi-organ nature. 
- As part of our research, we analyzed bone marrow histological features, molecular characteristics, and laboratory and clinical parameters at presentation in a large cohort of SM patients.

- $\quad$ The frequency of KIT D816V mutation in our study population was evaluated by SM subtype. The established occurrence of this particular mutation and its impact on the clinicohaematological findings was compliant with general literature data.

- $\quad$ Life expectancy of SM patients was compared to age- and sex-matched controls. In addition, the prognostic relevance of the 2008 WHO classification of SM in the investigated patient population was evaluated. Our analyses on Hungarian patients revealed that the survival in SM (including all subtypes) is worse than that expected in the age- and sex-matched Hungarian population. The life expectancy of patients with ISM was excellent whereas SM-AHNMD and ASM groups had a reduced median survival. The distribution of subtypes and the survival pattern seen in our SM study population was similar to that reported in the largest trial previously published.

- $\quad$ Epidemiological data on SM, an orphan Ph-negative MPN are only sparsely available in the literature. With our research, we provided important new data on SM and quantified its cumulative incidence in the South Great Plain region of Hungary.

\subsection{HYPEREOSINOPHILIC SYNDROME}

HES is a particularly heterogeneous entity with a wide range of clinical manifestations and a typically multi-organ presentation, facilitating a multidisciplinary thinking in its management

- We first performed investigations with 3DSTE, a novel non-invasive cardiac diagnostic tool in patients with HES. All HES patients and their age- and sex-matched healthy controls underwent complete 2D Doppler echocardiography and 3DSTE.

- $\quad$ On 3DSTE, increased LA volumes and LA-SVs were demonstrated in HES patients, accompanied by reduced LA-CS values. These findings suggest a structural and functional LA remodelling in the evaluated patients. The diagnostic work-up requires a close and effective multidisciplinary co-operation between the cardiologist, neurologist, the neuroradiologist, and the haematologist in order to achieve an early and precise diagnosis and a successful management of cases with hypereosinophilic syndrome.

The diagnosis and treatment of all three investigated Ph-negative MPDs is highly complex, calling for a multidisciplinary collaboration among several clinical and non-clinical divisions. 


\section{REFERENCES}

[1] Dameshek W: Some speculations on the myeloproliferative syndromes. Blood 1951;6(4): 372-375.

[2] Tefferi A: The history of myeloproliferative disorders: before and after Dameshek. Leukemia 2008;22(1): 3-13.

[3] Michiels JJ, Berneman Z, Schroyens W, De Raeve H: Changing concepts of diagnostic criteria of myeloproliferative disorders and the molecular etiology and classification of myeloproliferative neoplasms: from Dameshek 1950 to Vainchenker 2005 and beyond. Acta haematologica 2015;133(1): 36-51.

[4] Tefferi A, Gilliland DG: Oncogenes in myeloproliferative disorders. Cell cycle 2007;6(5): 550-566.

[5] Tefferi A, Thiele J, Vardiman JW: The 2008 World Health Organization classification system for myeloproliferative neoplasms: order out of chaos. Cancer 2009;115(17): 38423847.

[6] Tefferi A: Novel mutations and their functional and clinical relevance in myeloproliferative neoplasms: JAK2, MPL, TET2, ASXL1, CBL, IDH and IKZF1. Leukemia 2010;24(6): 1128-1138.

[7] Kleinfeldt T, Nienaber CA, Kische S, Akin I, Turan RG, Korber T, et al.: Cardiac manifestation of the hypereosinophilic syndrome: new insights. Clinical research in cardiology : official journal of the German Cardiac Society 2010;99(7): 419-427.

[8] Sen T, Gungor O, Akpinar I, Cetin M, Tufekcioglu O, Golbasi Z: Cardiac involvement in hypereosinophilic syndrome. Texas Heart Institute journal / from the Texas Heart Institute of St Luke's Episcopal Hospital, Texas Children's Hospital 2009;36(6): 628-629.

[9] Shah R, Ananthasubramaniam K: Evaluation of cardiac involvement in hypereosinophilic syndrome: complementary roles of transthoracic, transesophageal, and contrast echocardiography. Echocardiography 2006;23(8): 689-691.

[10] Moulard O, Mehta J, Fryzek J, Olivares R, Iqbal U, Mesa RA: Epidemiology of myelofibrosis, essential thrombocythemia, and polycythemia vera in the European Union. Eur J Haematol 2014;92(4): 289-297.

[11] Tefferi A, Rumi E, Finazzi G, Gisslinger H, Vannucchi AM, Rodeghiero F, et al.: Survival and prognosis among 1545 patients with contemporary polycythemia vera: an international study. Leukemia 2013;27(9): 1874-1881.

[12] Ania BJ, Suman VJ, Sobell JL, Codd MB, Silverstein MN, Melton LJ, 3rd: Trends in the incidence of polycythemia vera among Olmsted County, Minnesota residents, 1935-1989. American journal of hematology 1994;47(2): 89-93.

[13] Vardiman JW, Thiele J, Arber DA, Brunning RD, Borowitz MJ, Porwit A, et al.: The 2008 revision of the World Health Organization (WHO) classification of myeloid neoplasms and acute leukemia: rationale and important changes. Blood 2009;114(5): 937-951.

[14] Pardanani A, Lasho TL, Finke C, Hanson CA, Tefferi A: Prevalence and clinicopathologic correlates of JAK2 exon 12 mutations in JAK2V617F-negative polycythemia vera. Leukemia 2007;21(9): 1960-1963.

[15] Tefferi A, Guglielmelli P, Larson DR, Finke C, Wassie EA, Pieri L, et al.: Long-term survival and blast transformation in molecularly annotated essential thrombocythemia, polycythemia vera, and myelofibrosis. Blood 2014;124(16): 2507-2513; quiz 2615.

[16] Tefferi A: Polycythemia vera and essential thrombocythemia: 2012 update on diagnosis, risk stratification, and management. American journal of hematology 2012;87(3): 285-293. 
[17] Tefferi A, Barbui T: Polycythemia vera and essential thrombocythemia: 2015 update on diagnosis, risk-stratification and management. Am J Hematol 2015;90(2): 162-173.

[18] Tefferi A, Elliott M: Thrombosis in myeloproliferative disorders: prevalence, prognostic factors, and the role of leukocytes and JAK2V617F. Semin Thromb Hemost 2007;33(4): 313320 .

[19] Falanga A, Marchetti M: Thrombotic disease in the myeloproliferative neoplasms. Hematology Am Soc Hematol Educ Program 2012;2012: 571-581.

[20] Barbui T, Finazzi G, Falanga A: Myeloproliferative neoplasms and thrombosis. Blood 2013;122(13): 2176-2184.

[21] Tefferi A: Polycythemia vera and essential thrombocythemia: 2013 update on diagnosis, risk-stratification, and management. Am J Hematol 2013;88(6): 507-516.

[22] AB.Hill, ed. A short textbook of medical statistics. London: Hodder and Stoughton. 1984: 170.

[23] Baxter EJ, Scott LM, Campbell PJ, East C, Fourouclas N, Swanton S, et al.: Acquired mutation of the tyrosine kinase JAK2 in human myeloproliferative disorders. Lancet 2005;365(9464): 1054-1061.

[24] Benson EW, Hardy R, Chaffin C, Robinson CA, Konrad RJ: New automated chemiluminescent assay for erythropoietin. Journal of clinical laboratory analysis 2000;14(6): 271-273.

[25] Posfai E, Marton I, Kiss-Laszlo Z, Kotosz B, Szell M, Borbenyi Z: Thrombosis and risk factors in female patients with a rare acquired thrombophilia: chronic myeloproliferative disorder - polycythaemia vera and essential thrombocythaemia. Eur Rev Med Pharmacol Sci 2014;18(24): 3810-3818.

[26] Arock M, Valent P: Pathogenesis, classification and treatment of mastocytosis: state of the art in 2010 and future perspectives. Expert review of hematology 2010;3(4): 497-516.

[27] Lim KH, Tefferi A, Lasho TL, Finke C, Patnaik M, Butterfield JH, et al.: Systemic mastocytosis in 342 consecutive adults: survival studies and prognostic factors. Blood 2009;113(23): 5727-5736.

[28] Pardanani A: Systemic mastocytosis in adults: 2013 update on diagnosis, risk stratification, and management. Am J Hematol 2013;88(7): 612-624.

[29] Pardanani A: Systemic mastocytosis in adults: 2015 update on diagnosis, risk stratification, and management. Am J Hematol 2015;90(3): 250-262.

[30] Swerdlow SH, Campo, E., Harris, N.L., Jaffe, E.S., Pileri, S.A., Stein, H., Thiele, J., Vardiman, J.W, ed. WHO Classification of Tumours of Haematopoietic and Lymphoid Tissues. 2008: 439.

[31] Valent P, Akin C, Sperr WR, Mayerhofer M, Fodinger M, Fritsche-Polanz R, et al.: Mastocytosis: pathology, genetics, and current options for therapy. Leukemia \& lymphoma 2005;46(1): 35-48.

[32] Pardanani A: How I treat patients with indolent and smoldering mastocytosis (rare conditions but difficult to manage). Blood 2013;121(16): 3085-3094.

[33] Amon U, Hartmann K, Horny HP, Nowak A: Mastocytosis - an update. Journal der Deutschen Dermatologischen Gesellschaft = Journal of the German Society of Dermatology : JDDG 2010;8(9): 695-711; quiz 712.

[34] McCabe C, Claxton K, Tsuchiya A: Orphan drugs and the NHS: should we value rarity? BMJ 2005;331(7523): 1016-1019.

[35] Szegedi M, Molnar MJ, Boncz I, Kosztolanyi G: [Shift of focus in the financing of Hungarian drugs. Reimbursement for orphan drugs for treating rare diseases: financing of enzyme replacement therapy in Hungary]. Orv Hetil 2014;155(44): 1735-1741. 
[36] Valent P, Arock M, Bonadonna P, Brockow K, Broesby-Olsen S, Escribano L, et al.: European Competence Network on Mastocytosis (ECNM): 10-year jubilee, update, and future perspectives. Wiener klinische Wochenschrift 2012;124(23-24): 807-814.

[37] Valent P: Mastocytosis: a paradigmatic example of a rare disease with complex biology and pathology. American journal of cancer research 2013;3(2): 159-172.

[38] Cohen SS, Skovbo S, Vestergaard H, Kristensen T, Moller M, Bindslev-Jensen C, et al.: Epidemiology of systemic mastocytosis in Denmark. Br J Haematol 2014;166(4): 521-528.

[39] van Doormaal JJ, Arends S, Brunekreeft KL, van der Wal VB, Sietsma J, van Voorst Vader PC, et al.: Prevalence of indolent systemic mastocytosis in a Dutch region. The Journal of allergy and clinical immunology 2013;131(5): 1429-1431 e1421.

[40] Kirshenbaum AS, Metcalfe DD: Growth of human mast cells from bone marrow and peripheral blood-derived CD34+ pluripotent progenitor cells. Methods Mol Biol 2006;315: 105-112.

[41] Beaven MA: Our perception of the mast cell from Paul Ehrlich to now. Eur J Immunol 2009;39(1): 11-25.

[42] Nagata H, Worobec AS, Oh CK, Chowdhury BA, Tannenbaum S, Suzuki Y, et al.: Identification of a point mutation in the catalytic domain of the protooncogene c-kit in peripheral blood mononuclear cells of patients who have mastocytosis with an associated hematologic disorder. Proceedings of the National Academy of Sciences of the United States of America 1995;92(23): 10560-10564.

[43] Fritsche-Polanz R, Jordan JH, Feix A, Sperr WR, Sunder-Plassmann G, Valent P, et al.: Mutation analysis of C-KIT in patients with myelodysplastic syndromes without mastocytosis and cases of systemic mastocytosis. British journal of haematology 2001;113(2): 357-364.

[44] Orfao A, Garcia-Montero AC, Sanchez L, Escribano L, Rema: Recent advances in the understanding of mastocytosis: the role of KIT mutations. Br J Haematol 2007;138(1): 12-30. [45] Sotlar K, Colak S, Bache A, Berezowska S, Krokowski M, Bultmann B, et al.: Variable presence of KITD816V in clonal haematological non-mast cell lineage diseases associated with systemic mastocytosis (SM-AHNMD). The Journal of pathology 2010;220(5): 586-595.

[46] Valent P, Akin C, Escribano L, Fodinger M, Hartmann K, Brockow K, et al.: Standards and standardization in mastocytosis: consensus statements on diagnostics, treatment recommendations and response criteria. Eur J Clin Invest 2007;37(6): 435-453.

[47] Valent P, Sperr WR, Akin C: How I treat patients with advanced systemic mastocytosis. Blood 2010;116(26): 5812-5817.

[48] Lim KH, Pardanani A, Butterfield JH, Li CY, Tefferi A: Cytoreductive therapy in 108 adults with systemic mastocytosis: Outcome analysis and response prediction during treatment with interferon-alpha, hydroxyurea, imatinib mesylate or 2-chlorodeoxyadenosine. American journal of hematology 2009;84(12): 790-794.

[49] Pardanani A: Systemic mastocytosis in adults: 2013 update on diagnosis, risk stratification, and management. Am J Hematol 2013.

[50] Pardanani A, Tefferi A: Systemic mastocytosis in adults: a review on prognosis and treatment based on 342 Mayo Clinic patients and current literature. Current opinion in hematology 2010;17(2): 125-132.

[51] Valent P: Biology, classification and treatment of human mastocytosis. Wiener klinische Wochenschrift 1996;108(13): 385-397.

[52] Escribano L, Akin C, Castells M, Orfao A, Metcalfe DD: Mastocytosis: current concepts in diagnosis and treatment. Annals of hematology 2002;81(12): 677-690.

[53] Valent P, Akin C, Sperr WR, Horny HP, Arock M, Lechner K, et al.: Diagnosis and treatment of systemic mastocytosis: state of the art. British journal of haematology 2003;122(5): 695-717. 
[54] Imelda Marton Éva Pósfai, Zita Borbényi, Csaba Bödör, Papp Gergely, Demeter Judit, Irma Korom, Erika Varga, Zsuzsanna Bata-Csörgö: Therapeutic challenge during the longterm follow-up of a patient with indolent systemic mastocytosis with extensive cutaneous involvement. European Review for Medical and Pharmacological Sciences 2014. 2015;19(9):1607-9

[55] Marton I, Krenacs L, Bagdi E, Bakos A, Demeter J, Borbenyi Z: Clinical and Molecular Diagnostic Evaluation of Systemic Mastocytosis in the South-Eastern Hungarian Population Between 2001-2013 - A Single Centre Experience. Pathol Oncol Res 2016 Apr;22(2):293-9.

[56] Population census (2011)

Regional data - Bács-Kiskun county: http://www.ksh.hu/nepszamlalas/tables_regional_03;

Regional data - Békés county: http://www.ksh.hu/nepszamlalas/tables regional 04;

Rgional data - Csongrád county: http://www.ksh.hu/nepszamlalas/tables regional 06.

[57] Brigden M, Graydon C: Eosinophilia detected by automated blood cell counting in ambulatory North American outpatients. Incidence and clinical significance. Arch Pathol Lab Med 1997;121(9): 963-967.

[58] Rothenberg ME: Eosinophilia. N Engl J Med 1998;338(22): 1592-1600.

[59] Tefferi A, Patnaik MM, Pardanani A: Eosinophilia: secondary, clonal and idiopathic. $\mathrm{Br}$ J Haematol 2006;133(5): 468-492.

[60] Borbenyi Z: [Disorders with eosinophilia, treatment of hypereosinophilic syndrome]. Orv Hetil 2005;146(18 Suppl 1): 911-916.

[61] Valent P, Klion AD, Horny HP, Roufosse F, Gotlib J, Weller PF, et al.: Contemporary consensus proposal on criteria and classification of eosinophilic disorders and related syndromes. J Allergy Clin Immunol 2012;130(3): 607-612 e609.

[62] Savage N, George TI, Gotlib J: Myeloid neoplasms associated with eosinophilia and rearrangement of PDGFRA, PDGFRB, and FGFR1: a review. International journal of laboratory hematology 2013;35(5): 491-500.

[63] Cools J, DeAngelo DJ, Gotlib J, Stover EH, Legare RD, Cortes J, et al.: A tyrosine kinase created by fusion of the PDGFRA and FIP1L1 genes as a therapeutic target of imatinib in idiopathic hypereosinophilic syndrome. The New England journal of medicine 2003;348(13): 1201-1214.

[64] Gotlib J: World Health Organization-defined eosinophilic disorders: 2012 update on diagnosis, risk stratification, and management. Am J Hematol 2012;87(9): 903-914.

[65] Jovanovic JV, Score J, Waghorn K, Cilloni D, Gottardi E, Metzgeroth G, et al.: Lowdose imatinib mesylate leads to rapid induction of major molecular responses and achievement of complete molecular remission in FIP1L1-PDGFRA-positive chronic eosinophilic leukemia. Blood 2007;109(11): 4635-4640.

[66] Pardanani A, Brockman SR, Paternoster SF, Flynn HC, Ketterling RP, Lasho TL, et al.: FIP1L1-PDGFRA fusion: prevalence and clinicopathologic correlates in 89 consecutive patients with moderate to severe eosinophilia. Blood 2004;104(10): 3038-3045.

[67] Pardanani A, Ketterling RP, Li CY, Patnaik MM, Wolanskyj AP, Elliott MA, et al.: FIP1L1-PDGFRA in eosinophilic disorders: prevalence in routine clinical practice, long-term experience with imatinib therapy, and a critical review of the literature. Leuk Res 2006;30(8): 965-970.

[68] Chusid MJ, Dale DC, West BC, Wolff SM: The hypereosinophilic syndrome: analysis of fourteen cases with review of the literature. Medicine 1975;54(1): 1-27.

[69] Gotlib J: World Health Organization-defined eosinophilic disorders: 2014 update on diagnosis, risk stratification, and management. Am J Hematol 2014;89(3): 325-337.

[70] Gotlib J, Cools J, Malone JM, 3rd, Schrier SL, Gilliland DG, Coutre SE: The FIP1L1PDGFRalpha fusion tyrosine kinase in hypereosinophilic syndrome and chronic eosinophilic 
leukemia: implications for diagnosis, classification, and management. Blood 2004;103(8): 2879-2891.

[71] Fauci AS, Harley JB, Roberts WC, Ferrans VJ, Gralnick HR, Bjornson BH: NIH conference. The idiopathic hypereosinophilic syndrome. Clinical, pathophysiologic, and therapeutic considerations. Annals of internal medicine 1982;97(1): 78-92.

[72] Spry CJ, Davies J, Tai PC, Olsen EG, Oakley CM, Goodwin JF: Clinical features of fifteen patients with the hypereosinophilic syndrome. The Quarterly journal of medicine 1983;52(205): 1-22.

[73] Lefebvre C, Bletry O, Degoulet P, Guillevin L, Bentata-Pessayre M, Le Thi Huong D, et al.: [Prognostic factors of hypereosinophilic syndrome. Study of 40 cases]. Annales de medecine interne 1989;140(4): 253-257.

[74] Mannelli L, Cherian V, Nayar A, Srichai-Parsia M: Loeffler's endocarditis in hypereosinophilic syndrome. Curr Probl Diagn Radiol 2012;41(4): 146-148.

[75] Ogbogu PU, Rosing DR, Horne MK, 3rd: Cardiovascular manifestations of hypereosinophilic syndromes. Immunol Allergy Clin North Am 2007;27(3): 457-475.

[76] Moosbauer C, Morgenstern E, Cuvelier SL, Manukyan D, Bidzhekov K, Albrecht S, et al.: Eosinophils are a major intravascular location for tissue factor storage and exposure. Blood 2007;109(3): 995-1002.

[77] Wang JG, Mahmud SA, Thompson JA, Geng JG, Key NS, Slungaard A: The principal eosinophil peroxidase product, HOSCN, is a uniquely potent phagocyte oxidant inducer of endothelial cell tissue factor activity: a potential mechanism for thrombosis in eosinophilic inflammatory states. Blood 2006;107(2): 558-565.

[78] Nemes A, Kalapos A, Domsik P, Forster T: [Three-dimensional speckle-tracking echocardiography -- a further step in non-invasive three-dimensional cardiac imaging]. Orv Hetil 2012;153(40): 1570-1577.

[79] Kleijn SA, Aly MF, Terwee CB, van Rossum AC, Kamp O: Comparison between direct volumetric and speckle tracking methodologies for left ventricular and left atrial chamber quantification by three-dimensional echocardiography. Am J Cardiol 2011;108(7): 10381044.

[80] Nagaya M, Kawasaki M, Tanaka R, Onishi N, Sato N, Ono K, et al.: Quantitative validation of left atrial structure and function by two-dimensional and three-dimensional speckle tracking echocardiography: a comparative study with three-dimensional computed tomography. Journal of cardiology 2013;62(3): 188-194.

[81] Nemes A, Domsik P, Kalapos A, Lengyel C, Orosz A, Forster T: Comparison of threedimensional speckle tracking echocardiography and two-dimensional echocardiography for evaluation of left atrial size and function in healthy volunteers (results from the MAGYARHealthy study). Echocardiography 2014;31(7): 865-871.

[82] Domsik P, Kalapos A, Chadaide S, Sepp R, Hausinger P, Forster T, et al.: Threedimensional speckle tracking echocardiography allows detailed evaluation of left atrial function in hypertrophic cardiomyopathy--insights from the MAGYAR-Path Study. Echocardiography 2014;31(10): 1245-1252.

[83] Mochizuki A, Yuda S, Oi Y, Kawamukai M, Nishida J, Kouzu H, et al.: Assessment of left atrial deformation and synchrony by three-dimensional speckle-tracking echocardiography: comparative studies in healthy subjects and patients with atrial fibrillation. $J$ Am Soc Echocardiogr 2013;26(2): 165-174.

[84] Chadaide S, Domsik P, Kalapos A, Saghy L, Forster T, Nemes A: Three-dimensional speckle tracking echocardiography-derived left atrial strain parameters are reduced in patients with atrial fibrillation (results from the MAGYAR-path study). Echocardiography 2013;30(9): 1078-1083. 
[85] Nemes A, Kalapos A, Domsik P, Marton I, Borbenyi Z, Forster T: Three-dimensional speckle-tracking echocardiography in Loeffler endocarditis: case report from the MAGYARPath Study. Herz 2014;39(6): 722-724.

[86] Marton I, Posfai E, Annus JK, Borbenyi Z, Nemes A, Vecsei L, et al.: Watershed Infarction in Hypereosinophilic Syndrome: A Diagnostic Dilemma in Fip111-Pdgfr AlphaAssociated Myeloid Neoplasm. Ideggyogyaszati szemle 2015;68(5-6): 212-216.

[87] Landolfi R, Di Gennaro L, Falanga A: Thrombosis in myeloproliferative disorders: pathogenetic facts and speculation. Leukemia 2008;22(11): 2020-2028.

[88] Barbui T, Carobbio A, Rumi E, Finazzi G, Gisslinger H, Rodeghiero F, et al.: In contemporary patients with polycythemia vera, rates of thrombosis and risk factors delineate a new clinical epidemiology. Blood 2014;124(19): 3021-3023.

[89] Marchioli R, Finazzi G, Landolfi R, Kutti J, Gisslinger H, Patrono C, et al.: Vascular and neoplastic risk in a large cohort of patients with polycythemia vera. J Clin Oncol 2005;23(10): 2224-2232.

[90] Marchioli R, Finazzi G, Specchia G, Cacciola R, Cavazzina R, Cilloni D, et al.: Cardiovascular events and intensity of treatment in polycythemia vera. $N$ Engl $\mathrm{J} \mathrm{Med}$ 2013;368(1): 22-33.

[91] Zoltán Vokó , György Széles, László Kardos, Renáta Németh, Ádány R: The epidemiology of cerebrovascular diseases in Hungary after the millennium. LAM ( Lege Artis Medicinae) 2008;18(1):31-38.

[92] Barbui T, Finazzi G, Carobbio A, Thiele J, Passamonti F, Rumi E, et al.: Development and validation of an International Prognostic Score of thrombosis in World Health Organization-essential thrombocythemia (IPSET-thrombosis). Blood 2012;120(26): 51285133; quiz 5252.

[93] Crisa E, Venturino E, Passera R, Prina M, Schinco P, Borchiellini A, et al.: A retrospective study on 226 polycythemia vera patients: impact of median hematocrit value on clinical outcomes and survival improvement with anti-thrombotic prophylaxis and nonalkylating drugs. Ann Hematol 2010;89(7): 691-699.

[94] Vannucchi AM, Barbui T, Cervantes F, Harrison C, Kiladjian JJ, Kroger N, et al.: Philadelphia chromosome-negative chronic myeloproliferative neoplasms: ESMO Clinical Practice Guidelines for diagnosis, treatment and follow-up. Ann Oncol 2015;26 Suppl 5: v8599.

[95] Tefferi A, Barbui T: New and treatment-relevant risk stratification for thrombosis in essential thrombocythemia and polycythemia vera. Am J Hematol 2015;90(8): 683-685.

[96] Akin C: Clonality and molecular pathogenesis of mastocytosis. Acta Haematol 2005;114(1): 61-69.

[97] Escribano L, Alvarez-Twose I, Sanchez-Munoz L, Garcia-Montero A, Nunez R, Almeida J, et al.: Prognosis in adult indolent systemic mastocytosis: a long-term study of the Spanish Network on Mastocytosis in a series of 145 patients. J Allergy Clin Immunol 2009;124(3): 514-521.

[98] Yoshikawa H: Neuropathological findings in hypereosinophilic syndrome. Intern Med 2003;42(5): 381-382.

[99] Grigoryan M, Geisler SD, St Louis EK, Baumbach GL, Davis PH: Cerebral arteriolar thromboembolism in idiopathic hypereosinophilic syndrome. Arch Neurol 2009;66(4): 528531.

[100] Sethi HS, Schmidley JW: Cerebral infarcts in the setting of eosinophilia: three cases and a discussion. Arch Neurol 2010;67(10): 1275-1277. 


\section{ACKNOWLEDGEMENTS}

I wish to express my sincere gratitude to my tutors Prof. Zita Borbényi M.D., Ph.D. and Prof. Attila Nemes M.D., Ph.D., D.Sc. for their continuous support and advice for my doctoral studies. I am indebted to them for their constant guidance, patience, and motivation as well as the valuable discussions during my work.

Due to the multidisciplinary nature of my research, I was favoured to establish a fruitful collaboration with a number of excellent clinicians of partner disciplines at the University of Szeged. As such, I am especially thankful to Katalin Sas M.D., Ph.D. and János Annus M.D. for their supervision in neurology, to Erika Vörös M.D., Ph.D. for her valuable consultation in neuroradiology, to Prof. Zsuzsanna Bata-Csörgő M.D., Ph.D., D.Sc. for her shared expertise and guidance in dermatology, as well as to Anita Kalapos M.D. and Péter Domsik M.D., Ph.D. for their cooperation in cardiology.

I wish to extend my sincerest thanks to the outstanding representatives of diagnostic disciplines for their expert engagement with my research, in particular to Prof. László Krenács M.D.,Ph.D., D.Sc. and Enikő Bagdy M.D., Ph.D. and Annamária Bakos M.D. from the Laboratory of Tumour Pathology and Molecular Diagnostics, Szeged.

I am most grateful to Prof. Márta Széll Ph.D., D.Sc.and Zsuzsanna László Ph.D. from the Institute of Medical Genetics, University of Szeged for their active involvement in my studies. I also wish to thank the collaboration to Hajnalka Andrikovics M.D., Ph.D from the Hungarian National Blood Transfusion Service Laboratory, Budapest and Csaba Bödör Ph.D, D.Sc., the $1^{\text {st }}$ Department of Pathology and Experimental Cancer Research, Semmelweis University, Budapest.

Moreover, I wish to express my appreciation and thanks to Balázs Kotosz Ph.D. statistician for his professional support with the analysis of data and the validation of results as well as to Prof. Zoltán Vokó for his supervision in epidemiology.

I am greatly thankful to Éva Pósfai M.D. and my co-authors and all my colleagues from the Division of Haematology, $2^{\text {nd }}$ Department of Internal Medicine and Cardiology Centre, University of Szeged, who gave me huge support.

And last but not least, I wish to thank the endless patience and encouragement of my husband and sons who enabled me to accomplish this work and my parents who have never stopped in believing in me. 